$\underline{\text { Preprint typeset in JHEP style - PAPER VERSION }}$

DFPD 03/TH/38

\title{
4-point effective actions in open and closed superstring theory
}

\author{
Osvaldo Chandía \\ Nucleare, Sezione di Padova, Italia \\ E-mail: chandia@pd.infn.it. \\ Ricardo Medina \\ Instituto de Ciências, Universidade Federal de Itajubá \\ Itajubá, Minas Gerais, Brazil \\ E-mail: rmedina@unifei.edu.br.
}

Dipartamento de Fisica, Università degli Studi di Padova and Istituto Nazionale di Fisica

\begin{abstract}
Recently the effective action for the 4-point functions in abelian open superstring theory has been derived, giving an explicit construction of the bosonic and fermionic terms of this infinite $\alpha^{\prime}$ series. In the present work we generalize this result to the nonabelian case. We test our result, at $\alpha^{\prime 3}$ and $\alpha^{\prime 4}$ order, with several existing versions for these terms, finding agreement in most of the cases. We also apply these ideas to derive the effective action for the 4-point functions of the NS-NS sector of closed superstring theory, to all order in $\alpha^{\prime}$.
\end{abstract}




\section{Contents}

1. Introduction 1

2. Nonabelian open superstring 4-point effective action 3

2.1 Review of the abelian effective action 3

2.2 The nonabelian formula 4

2.3 Linear Supersymmetry 5

$\begin{array}{lll}2.4 & \alpha^{\prime} \text { expansion } & 7\end{array}$

$2.5 \mathcal{O}\left(\alpha^{\prime 3}\right)$ and $\mathcal{O}\left(\alpha^{\prime 4}\right)$ tests 8

2.6 Higher order terms in the effective action 9

3. Closed superstring 4-point effective action $\quad 10$

3.1 The general formula 10

3.2 Higher order terms in the effective action 12

4. Final remarks and conclusions $\quad 13$

A. Conventions and Identities $\quad 14$

B. Derivation of the 4-particle scattering amplitudes from the effective action 15

B.1 4-particle scattering amplitudes in SYM 16

$\begin{array}{ll}\text { B.2 The 4-point effective action } & 18\end{array}$

$\begin{array}{ll}\text { B.3 The scattering amplitudes } & 19\end{array}$

C. $f$ - $f$ part of the $\mathcal{O}\left(\alpha^{\prime 5}\right)$ action 20

\section{Introduction}

The subject of the string $\alpha^{\prime}$ corrections to the Maxwell and Yang-Mills lagrangians, in its bosonic as well as in its supersymmetric generalization, began a long time ago. The first striking result consisted in the discovery of the Born-Infeld lagrangian [1] as describing the abelian effective theory of interacting open massless strings [2]. This result contains the infinite $\alpha^{\prime}$ series of corrections to the Maxwell lagrangian, but it does not include the derivative terms of the series. Since then, the nonabelian $\alpha^{\prime 2} F^{4}$ terms $[3,4]$ the nonabelian $\alpha^{\prime 3} D^{2} F^{4}$ terms $[5]^{1}$ and the abelian $\alpha^{\prime 4} \partial^{4} F^{4}$ terms [6] were determined, among other things. All these results were derived using the $\sigma$-model or the scattering amplitude approach.

In the last decade new interest arose in this subject since the discovery that the low energy dynamics of open superstrings captured an equivalent description in terms of D-branes [7]. Some time afterwards, the supersymmetric generalization of the Born-Infeld lagrangian was constructed $[8,9,10,11]$.

Up to now, including the previous results in [2] and [11], infinite $\alpha^{\prime}$ series for low energy effective actions have only been obtained in the abelian case $[12,13]$. Besides the attempt of proposing a

\footnotetext{
${ }^{1}$ This work also included some partial information of the $\alpha^{3} F^{5}$ terms.
} 
symmetrized trace prescription as a nonabelian generalization of the Born-Infeld lagrangian [14], in the nonabelian case an analogue result is not known and all the information that has been derived is strictly perturbative in $\alpha^{\prime}$. In this context, the scattering amplitude approach has been used to derive boson-boson, boson-fermion and fermion-fermion terms of the effective action which are only quartic in the fields $[15,16]$, since the general wisdom of scattering amplitudes in string theory has traditionally gone up to 4-point amplitudes [17] only. Indeed, a complete 5-point amplitude has only been derived for the open superstring in the past year $[18]^{2}$. It has been used to confirm not only the nonabelian $\alpha^{\prime 3} D^{2} F^{4}$ terms, but also the nonabelian $\alpha^{\prime 3} F^{5}$ terms [19], in which some controversy went on $[5,20,19]$.

In the last three years, several successful methods arose as an alternative way to derive the $\alpha^{\prime}$ corrections to the Super Yang-Mills (SYM) lagrangian. This includes: the determination of the 1-loop effective action for $N=4 \mathrm{SYM}[20,21]$; the requirement of certain BPS solutions to the deformed equations of motion [22, 19, 23]; the supersymmetric deformations to the SYM and the Super Maxwell lagrangians [24, 25] and the construction of supersymmetric invariants by different superspace techniques $[26,27,28]$. Some of these methods were even used up to $\alpha^{\prime 4}$ level [23, 28], but in spite of their success in reproducing the open superstring effective action, all of them required in some moment some knowledge of the superstring 4-point amplitudes, to fix some undetermined coefficients.

Recently a new type of infinite $\alpha^{\prime}$ series contribution to the effective action has been derived [29]. It consists in the determination of the abelian supersymmetric terms which are sensible to 4-point amplitudes, at any order in $\alpha^{\prime}$.

In the present work we generalize this previous result to the nonabelian case. The infinite $\alpha^{\prime}$ corrections to the SYM action basically consists in a succession of nonabelian four field terms, all derived from the correponding ones in the $\mathcal{O}\left(\alpha^{\prime 2}\right)$ SYM invariant [26, 27] by taking appropriate derivatives to it, in direct analogy to the construction in [29].

We also include in this work the 4-point effective action for the NS-NS sector of closed superstring theory, which is also an infinite $\alpha^{\prime}$ series result derived along the same lines of [29]. In the closed superstring case, the determination of higher $\alpha^{\prime}$ corrections is much more involved than the corresponding ones in the open superstring case. The first type of $\alpha^{\prime}$ corrections found for closed superstrings consisted in the derivation of the $\alpha^{\prime 3} R^{4}$ terms in the gravitational sector of type II theories $[4,30]$. Subsequently, a similar analysis was done for the heterotic superstring $[31,32,33]$. It has been noted that there are no $\alpha^{\prime 4}$ corrections to the (tree level) 4-point effective action in the case of closed superstrings $[34]^{3}$. Some higher derivative terms have also been studied in $[35,36]$. Our paper is organized as follows. In section 2 we deal with the nonabelian generalization of [29]. We include a brief analysis about the linear supersymmetry of the effective action, which is possible to be constructed in the abelian case [29], but not in the nonabelian one, if only knowledge of 4-point amplitudes is to be considered. We have also included ${\alpha^{\prime}}^{3}$ and partial $\alpha^{4}$ tests of our action, together with the determination of the $\mathcal{O}\left(\alpha^{\prime 5}\right)$ terms, as an application. We do not comment about non linear supersymmetry in this work. In section 3 we apply the ideas of [29] to determine the effective action for the 4-point functions of the NS-NS sector of closed superstring theory. We confirm that this tree level action does not contain $\mathcal{O}\left(\alpha^{\prime 4}\right)$ terms [34], and we also derive some higher order $\alpha^{\prime}$ terms of it. Some final remarks and conclusions are given in section 4 .

\footnotetext{
${ }^{2}$ In [5] a partial computation of this 5-point amplitude was done.

${ }^{3}$ We thank R. Bentin for calling our attention to this point.
} 


\section{Nonabelian open superstring 4-point effective action}

\subsection{Review of the abelian effective action}

In [29] a tree level effective action was proposed for the 4-point functions of the abelian open superstring. In our conventions (see Appendix A) it is given by ${ }^{4}$

$$
\begin{aligned}
S_{\mathrm{eff}}^{\text {abel. }}= & \int d^{10} x\left[\left\{-\frac{1}{4} F_{\mu \nu} F^{\mu \nu}+\frac{i}{2} \bar{\psi} \gamma^{\mu} \partial_{\mu} \psi\right\}\right. \\
& -g^{2}{\alpha^{\prime}}^{2} \iiint \int\left\{\prod_{j=1}^{4} d^{10} x_{j} \delta^{(10)}\left(x-x_{j}\right)\right\} \\
& \mathcal{G}\left(\partial_{1} \cdot \partial_{2}+\partial_{3} \cdot \partial_{4}, \partial_{1} \cdot \partial_{4}+\partial_{2} \cdot \partial_{3}, \partial_{1} \cdot \partial_{3}+\partial_{2} \cdot \partial_{4}\right) \\
& \times\left\{F_{\mu \nu}\left(x_{1}\right) F^{\nu \rho}\left(x_{2}\right) F_{\rho \sigma}\left(x_{3}\right) F^{\sigma \mu}\left(x_{4}\right)-\frac{1}{4} F_{\mu \nu}\left(x_{1}\right) F^{\mu \nu}\left(x_{2}\right) F_{\rho \sigma}\left(x_{3}\right) F^{\rho \sigma}\left(x_{4}\right)\right. \\
& +2 i \bar{\psi}\left(x_{1}\right) \gamma^{\nu} \partial_{\rho} \psi\left(x_{2}\right) F_{\nu \mu}\left(x_{3}\right) F^{\mu \rho}\left(x_{4}\right)-i \bar{\psi}\left(x_{1}\right) \gamma^{\mu \nu \rho} \partial^{\sigma} \psi\left(x_{2}\right) F_{\mu \nu}\left(x_{3}\right) F_{\rho \sigma}\left(x_{4}\right) \\
& \left.\left.-\frac{1}{3} \bar{\psi}\left(x_{1}\right) \gamma_{\mu} \partial_{\nu} \psi\left(x_{2}\right) \bar{\psi}\left(x_{3}\right) \gamma^{\mu} \partial^{\nu} \psi\left(x_{4}\right)\right\}\right]
\end{aligned}
$$

where the term in brackets, in the first line of (2.1), is the $D=10$ Super Maxwell lagrangian and

$$
\mathcal{G}(s, t, u)=\frac{\Gamma\left(-\alpha^{\prime} s\right) \Gamma\left(-\alpha^{\prime} t\right)}{\Gamma\left(1-\alpha^{\prime} s-\alpha^{\prime} t\right)}+\frac{\Gamma\left(-\alpha^{\prime} t\right) \Gamma\left(-\alpha^{\prime} u\right)}{\Gamma\left(1-\alpha^{\prime} t-\alpha^{\prime} u\right)}+\frac{\Gamma\left(-\alpha^{\prime} u\right) \Gamma\left(-\alpha^{\prime} s\right)}{\Gamma\left(1-\alpha^{\prime} u-\alpha^{\prime} s\right)} .
$$

Note that $\mathcal{G}(s, t, u)$ is a symmetric function of $s, t$ and $u$.

In $[29]$ it was confirmed that

1. The 4-photon scattering amplitude derived from $S_{\text {eff }}^{\text {abel. }}$, in (2.1), reproduces the corresponding tree level amplitude derived in abelian Open Superstring theory:

$$
\mathcal{A}^{(4)}=16 i g^{2} \alpha^{\prime 2}(2 \pi)^{10} \delta^{(10)}\left(k_{1}+k_{2}+k_{3}+k_{4}\right) \mathcal{G}(s, t, u) K_{4 \mathrm{~b}},
$$

where

$$
s=-2 k_{1} \cdot k_{2}, \quad t=-2 k_{1} \cdot k_{4}, \quad u=-2 k_{1} \cdot k_{3},
$$

are the Mandelstam variables and $K_{4 \mathrm{~b}}$ is the 4-boson kinematic factor, given in eq.(B.4) of Appendix B.1. The Mandelstam variables satisfy the relation

$$
s+t+u=0 .
$$

2. The action is supersymmetric at every order in $\alpha^{\prime}$.

The main idea considered in [29] for proposing (2.1) as the abelian 4-point effective action was basically to make the correspondence

$$
k_{j}^{\mu} \rightarrow-i \frac{\partial}{\partial x_{\mu}^{j}} \quad(j=1,2,3,4 .),
$$

in a symmetrized way, in the momentum dependent factor $\mathcal{G}(s, t, u)$ that comes in the scattering amplitude (2.3), and that then goes in the effective action (2.1). This symmetrized prescription consists in first writing the Mandelstam variables as

$$
s=-k_{1} \cdot k_{2}-k_{3} \cdot k_{4}, \quad t=-k_{1} \cdot k_{4}-k_{2} \cdot k_{3} \text { and } u=-k_{1} \cdot k_{3}-k_{2} \cdot k_{4},
$$

\footnotetext{
${ }^{4}$ We use the notation $\partial_{k} \cdot \partial_{j}=\frac{\partial}{\partial x_{k}^{\mu}} \frac{\partial}{\partial x_{j \mu}}$.
} 
which are on-shell equivalent to (2.4), and then using the correspondence (2.6) as indicated above. As a result, $\mathcal{G}(s, t, u)$ becomes the differential operator $\mathcal{G}\left(\partial_{1} \cdot \partial_{2}+\partial_{3} \cdot \partial_{4}, \partial_{1} \cdot \partial_{4}+\partial_{2} \cdot \partial_{3}, \partial_{1} \cdot \partial_{3}+\partial_{2} \cdot \partial_{4}\right)$ appearing in (2.1), which is completely symmetric in the space-time coordinates. This simplifies the proof of the supersymmetry of (2.1), to every order in $\alpha^{\prime}$, as was seen in [29]. We will comment more about the issue of Supersymmetry in subsection 2.3.

\subsection{The nonabelian formula}

We now present the main result of section 2 , which is our nonabelian generalization of the effective action in (2.1):

$$
S_{\mathrm{eff}}^{\text {nonabel. }}=S_{\mathrm{SYM}}+S_{\alpha^{\prime} \text { corr. }}^{\text {nonabel. }},
$$

where

$$
S_{\mathrm{SYM}}=\int d^{10} x \operatorname{tr}\left\{-\frac{1}{4} F_{\mu \nu} F^{\mu \nu}+\frac{i}{2} \bar{\psi} \gamma^{\mu} D_{\mu} \psi\right\}
$$

is the $\mathrm{D}=10$ Super Yang-Mills action and

$$
\begin{aligned}
S_{\alpha^{\prime} \text { corr. }}^{\text {nonabel. }}= & -\frac{1}{2} g^{2}{\alpha^{\prime}}^{2} \int d^{10} x \iiint \int\left\{\prod_{j=1}^{4} d^{10} x_{j} \delta^{(10)}\left(x-x_{j}\right)\right\} \\
& \times \mathcal{G}_{\alpha^{\prime} \text { corr. }}^{a_{1} a_{2} a_{3} a_{4}}\left(D_{1} \cdot D_{2}+D_{3} \cdot D_{4}, D_{1} \cdot D_{4}+D_{2} \cdot D_{3}, D_{1} \cdot D_{3}+D_{2} \cdot D_{4}\right) \\
& \times\left[F_{a_{1} \mu \nu}\left(x_{1}\right) F_{a_{2}}^{\nu \rho}\left(x_{2}\right) F_{a_{3} \rho \sigma}\left(x_{3}\right) F_{a_{4}}^{\sigma \mu}\left(x_{4}\right)-\frac{1}{4} F_{a_{1} \mu \nu}\left(x_{1}\right) F_{a_{2}}^{\mu \nu}\left(x_{2}\right) F_{a_{3} \rho \sigma}\left(x_{3}\right) F_{a_{4}}^{\rho \sigma}\left(x_{4}\right)\right. \\
& +2 i \bar{\psi}_{a_{1}}\left(x_{1}\right) \gamma^{\nu} D_{\rho} \psi\left(x_{2}\right)_{a_{2}} F_{a_{3} \nu \mu}\left(x_{3}\right) F_{a_{4}}^{\mu \rho}\left(x_{4}\right)-i \bar{\psi}_{a_{1}}\left(x_{1}\right) \gamma^{\mu \nu \rho} D^{\sigma} \psi\left(x_{2}\right)_{a_{2}} F_{a_{3} \mu \nu}\left(x_{3}\right) F_{a_{4} \rho \sigma}\left(x_{4}\right) \\
& \left.-\frac{1}{3} \bar{\psi}_{a_{1}}\left(x_{1}\right) \gamma_{\mu} D_{\nu} \psi\left(x_{2}\right)_{a_{2}} \bar{\psi}_{a_{3}}\left(x_{3}\right) \gamma^{\mu} D^{\nu} \psi\left(x_{4}\right)_{a_{4}}\right]
\end{aligned}
$$

represents the piece of the effective action that contains the infinite $\alpha^{\prime}$ corrections that come from superstring theory.

The function $\mathcal{G}_{\alpha^{\prime} \text { corr. }}^{a_{1} a_{2} a_{3} a_{4}}$ appearing in (2.10) is given by

$$
\begin{aligned}
\mathcal{G}_{\alpha^{\prime} \text { corr. }}^{a_{1} a_{2} a_{3} a_{4}}(s, t, u)=[ & \left\{\operatorname{tr}\left(\lambda^{a_{1}} \lambda^{a_{2}} \lambda^{a_{3}} \lambda^{a_{4}}\right)+\operatorname{tr}\left(\lambda^{a_{4}} \lambda^{a_{3}} \lambda^{a_{2}} \lambda^{a_{1}}\right)\right\} f(s, t) \\
& +\left\{\operatorname{tr}\left(\lambda^{a_{1}} \lambda^{a_{3}} \lambda^{a_{2}} \lambda^{a_{4}}\right)+\operatorname{tr}\left(\lambda^{a_{4}} \lambda^{a_{2}} \lambda^{a_{3}} \lambda^{a_{1}}\right)\right\} f(t, u) \\
& \left.+\left\{\operatorname{tr}\left(\lambda^{a_{1}} \lambda^{a_{2}} \lambda^{a_{4}} \lambda^{a_{3}}\right)+\operatorname{tr}\left(\lambda^{a_{3}} \lambda^{a_{4}} \lambda^{a_{2}} \lambda^{a_{1}}\right)\right\} f(u, s)\right]
\end{aligned}
$$

where

$$
f(s, t)=\frac{\Gamma\left(-\alpha^{\prime} s\right) \Gamma\left(-\alpha^{\prime} t\right)}{\Gamma\left(1-\alpha^{\prime} s-\alpha^{\prime} t\right)}-\frac{1}{\alpha^{\prime 2} s t} .
$$

In (2.12) we have subtracted the poles to the Gamma factors since this last contribution has been considered separately in the SYM action in (2.8).

Note that, after substituting the symmetrized Mandelstam variables $(2.7), \mathcal{G}_{\alpha^{\prime} \text { corr. }}^{a_{1} a_{2} a_{3} a_{4}}(s, t, u)$ is symmetric in the pairs $\left(k_{j}, a_{j}\right)$.

A convenient way of writing the $\alpha^{\prime}$ expansion of $\mathcal{G}_{\alpha^{\prime} \text { corr. }}^{a_{1} a_{2} a_{3} a_{4}}$ will be commented in section 2.4 , to be used afterwards in (2.10).

As shown in Appendix B, the action in (2.10) indeed reproduces the 4-particle scattering amplitudes 
(at tree level) of nonabelian Open Superstring theory [17]: 4-boson, 2-boson/2-fermion and 4fermion amplitudes ${ }^{5}$ :

$$
\begin{array}{rl}
\mathcal{A}^{(4)}=8 & i g^{2} \alpha^{\prime 2}(2 \pi)^{10} \delta^{10}\left(k_{1}+k_{2}+k_{3}+k_{4}\right) \times \\
\times[ & \frac{\Gamma\left(-\alpha^{\prime} s\right) \Gamma\left(-\alpha^{\prime} t\right)}{\Gamma\left(1-\alpha^{\prime} s-\alpha^{\prime} t\right)}\left\{\operatorname{tr}\left(\lambda^{a_{1}} \lambda^{a_{2}} \lambda^{a_{3}} \lambda^{a_{4}}\right)+\operatorname{tr}\left(\lambda^{a_{4}} \lambda^{a_{3}} \lambda^{a_{2}} \lambda^{a_{1}}\right)\right\} \\
& +\frac{\Gamma\left(-\alpha^{\prime} t\right) \Gamma\left(-\alpha^{\prime} u\right)}{\Gamma\left(1-\alpha^{\prime} t-\alpha^{\prime} u\right)}\left\{\operatorname{tr}\left(\lambda^{a_{1}} \lambda^{a_{3}} \lambda^{a_{2}} \lambda^{a_{4}}\right)+\operatorname{tr}\left(\lambda^{a_{4}} \lambda^{a_{2}} \lambda^{a_{3}} \lambda^{a_{1}}\right)\right\} \\
& \left.+\frac{\Gamma\left(-\alpha^{\prime} u\right) \Gamma\left(-\alpha^{\prime} s\right)}{\Gamma\left(1-\alpha^{\prime} u-\alpha^{\prime} s\right)}\left\{\operatorname{tr}\left(\lambda^{a_{1}} \lambda^{a_{2}} \lambda^{a_{4}} \lambda^{a_{3}}\right)+\operatorname{tr}\left(\lambda^{a_{3}} \lambda^{a_{4}} \lambda^{a_{2}} \lambda^{a_{1}}\right)\right\}\right] \cdot K^{(4)}
\end{array}
$$

where $K^{(4)}$ is the kinematic factor associated to the corresponding scattering process.

Two immediate tests of (2.10) are the following:

1. Abelian limit:

Using (2.5) we see that in this limit (2.10) leads directly to (2.1).

2. $\alpha^{\prime 2}$ terms:

Using that the momentum dependent factor in (2.12) behaves as $f(s, t)=-\pi^{2} / 6+\mathcal{O}\left(\alpha^{\prime}\right)$, it can easily be seen that the first superstring correction to the Super Yang-Mills action is given by

$$
\begin{aligned}
S_{(2)}^{\text {nonabel. }}= & \frac{\pi^{2}}{2} g^{2} \alpha^{\prime 2} \int d^{10} x \operatorname{str}\left\{F_{\mu \nu} F^{\nu \rho} F_{\rho \sigma} F^{\sigma \mu}-\frac{1}{4} F_{\mu \nu} F^{\mu \nu} F_{\rho \sigma} F^{\rho \sigma}\right. \\
& \left.+2 i \bar{\psi} \gamma^{\nu} D_{\rho} \psi F_{\nu \mu} F^{\mu \rho}-i \bar{\psi} \gamma^{\mu \nu \rho} D^{\sigma} \psi F_{\mu \nu} F_{\rho \sigma}-\frac{1}{3} \bar{\psi} \gamma_{\mu} D_{\nu} \psi \bar{\psi} \gamma^{\mu} D^{\nu} \psi\right\},
\end{aligned}
$$

where "str" means the symmetrized trace[14], calculated as

$$
\begin{aligned}
\operatorname{str}\left(\lambda^{a_{1}} \lambda^{a_{2}} \lambda^{a_{3}} \lambda^{a_{4}}\right)= & \frac{1}{6}\left\{\operatorname{tr}\left(\lambda^{a_{1}} \lambda^{a_{2}} \lambda^{a_{3}} \lambda^{a_{4}}\right)+\operatorname{tr}\left(\lambda^{a_{1}} \lambda^{a_{4}} \lambda^{a_{3}} \lambda^{a_{2}}\right)+\operatorname{tr}\left(\lambda^{a_{1}} \lambda^{a_{4}} \lambda^{a_{2}} \lambda^{a_{3}}\right)\right. \\
& \left.+\operatorname{tr}\left(\lambda^{a_{1}} \lambda^{a_{3}} \lambda^{a_{2}} \lambda^{a_{4}}\right)+\operatorname{tr}\left(\lambda^{a_{1}} \lambda^{a_{3}} \lambda^{a_{4}} \lambda^{a_{2}}\right)+\operatorname{tr}\left(\lambda^{a_{1}} \lambda^{a_{2}} \lambda^{a_{4}} \lambda^{a_{3}}\right)\right\}
\end{aligned}
$$

The terms in (2.14) match completely with the corresponding ones of $[15]^{6}$ and are equivalent to the ones first derived in $[26,27]^{7}$.

We end this subsection recalling that, since the action (2.10) is a $\mathrm{D}=10$ Super Yang-Mills extension, the $\lambda^{a} U(N)$ generators are all in the adjoint representation, $\left(\lambda^{a}\right)^{b c}=-i f^{a b c}$, and therefore the six trace terms in (2.11), (2.13) and (2.15) satisfy $\operatorname{tr}\left(\lambda^{a} \lambda^{b} \lambda^{c} \lambda^{d}\right)=\operatorname{tr}\left(\lambda^{d} \lambda^{c} \lambda^{b} \lambda^{a}\right)$, reducing its number to only three.

\subsection{Linear Supersymmetry}

In the abelian case (2.1) the linear supersymmetry of the effective action was proved at all orders in $\alpha^{\prime}$ by arguing that

\footnotetext{
${ }^{5}$ Amplitudes involving an odd number of fermions are trivially null.

${ }^{6}$ See eq. (4.1) of this reference.

${ }^{7}$ The authors of [15] checked there that their $\alpha^{\prime 2}$ quartic terms coincide, up to on-shell terms and total derivatives, with the ones of $[26,27]$.
} 
1. The $\alpha^{\prime 2}$ order lagrangian, $\mathcal{L}_{(2)}^{a b e l}$, is supersymmetric.

2. The higher $\alpha^{\prime}$ lagrangians are automatically supersymmetric since they are constructed by the action of the differential operator $\mathcal{G}\left(\partial_{1} \cdot \partial_{2}+\partial_{3} \cdot \partial_{4}, \partial_{1} \cdot \partial_{4}+\partial_{2} \cdot \partial_{3}, \partial_{1} \cdot \partial_{3}+\partial_{2} \cdot \partial_{4}\right)$ on the nonlocal version of $\mathcal{L}_{(2)}^{\text {abel. }}, \mathcal{G}$ being symmetric in the spacetime coordinates.

Now, the difference between our nonabelian action (2.10) with the abelian one (2.1) is basically the covariantization of the terms. In the nonabelian case some care must be taken with the supersymmetry since, already at $\alpha^{\prime 2}$ order, additional terms should be considered [27]. The complete $\mathcal{L}_{(2)}^{\text {non abel. }}$ lagrangian is given, in our notation, by [27]

$$
\begin{aligned}
\mathcal{L}_{(2)}^{\text {non abel. }}= & \frac{\pi^{2}}{2} g^{2} \alpha^{\prime 2} \operatorname{str}\left\{F_{\mu \nu} F^{\nu \rho} F_{\rho \sigma} F^{\sigma \mu}-\frac{1}{4} F_{\mu \nu} F^{\mu \nu} F_{\rho \sigma} F^{\rho \sigma}\right. \\
& +2 i \bar{\psi} \gamma^{\nu} D_{\rho} \psi F_{\nu \mu} F^{\mu \rho}-i \bar{\psi} \gamma^{\mu \nu \rho} D^{\sigma} \psi F_{\mu \nu} F_{\rho \sigma}-\frac{1}{3} \bar{\psi} \gamma_{\mu} D_{\nu} \psi \bar{\psi} \gamma^{\mu} D^{\nu} \psi \\
& \left.-\frac{7}{60} g F^{\mu \nu} \bar{\psi} \gamma_{\mu \nu \rho} \psi\left\{\bar{\psi}, \gamma^{\rho} \psi\right\}+\frac{1}{360} g F^{\mu \nu} \bar{\psi} \gamma^{\rho \sigma \tau} \psi\left\{\bar{\psi}, \gamma_{\mu \nu \rho \sigma \tau} \psi\right\}\right\}
\end{aligned}
$$

The additional terms in the third line of (2.16), which we will abbreviate as $F(\psi \gamma \psi)^{2}$, are needed to form a nonabelian supersymmetric invariant, and they are only sensible to 5 and 6 -point amplitudes. It is tempting to include them nonlocally in our nonabelian action (2.10) in order to have, at any order in $\alpha^{\prime}$, a completely supersymmetric invariant action. We explore this possibility in the following lines concluding that this would only work at $\alpha^{\prime 2}$ order.

The linear supersymmetry transformations are given by

$$
\delta A_{\mu}=2 \bar{\epsilon} \gamma_{\mu} \psi, \quad \delta \psi=F_{\mu \nu}\left(\gamma^{\mu \nu} \epsilon\right)
$$

When analyzing the variation of (2.10) under the supersymmetric transformations (2.17), we may write this variation as a sum of two contributions:

1. The one that considers the variation of the nonlocal lagrangian (which now includes the $F(\psi \gamma \psi)^{2}$ terms $)$.

2. The one that considers the variation of

$$
\mathcal{G}_{\alpha^{\prime} \text { corr. }}^{a_{1} a_{2} a_{3} a_{4}}\left(D_{1} \cdot D_{2}+D_{3} \cdot D_{4}, D_{1} \cdot D_{4}+D_{2} \cdot D_{3}, D_{1} \cdot D_{3}+D_{2} \cdot D_{4}\right) \text {. }
$$

In the first situation, the piece containing the variation of only four field terms will vanish if we consider the symmetry of the $\mathcal{G}_{\alpha^{\prime} \text { corr. }}^{a_{1} a_{2} a_{3} a_{4}}$ operator, in direct analogy to the analysis done in [29] for the abelian case. But the piece containing the variation of the $F(\psi \gamma \psi)^{2}$ terms and the $\delta A_{\mu}$ variations from the covariant derivative terms, will also cancel. It is known that their sum cancels in the local lagrangian case [27], but it is possible to prove that in the nonlocal case they cancel as well. This can be seen as follows:

The nonlocal variation of the $F(\psi \gamma \psi)^{2}$ terms can generically be written as

$$
\epsilon F_{1} F_{4} \psi_{2}\{\psi, \psi\}_{3}
$$

and the corresponding generic nonlocal $\delta A_{\mu}$ variation (from the covariant derivative terms) has the form

$$
\epsilon F_{1} F_{2} \psi_{3}\{\psi, \psi\}_{4} \text {. }
$$

In (2.18) and (2.19) we use $F_{1}$, to denote $F_{a_{1}}\left(x_{1}\right)$, etc. and the spacetime indexes have been omitted since for all kinds of spacetime index contractions the argument will be the same. It is clear that 
both terms will combine since we can rename $2 \rightarrow 4,3 \rightarrow 2,4 \rightarrow 3$ in the second term and then the resultant combination will vanish, as it happens in the local case.

So, what would only remain to see is that the variation coming from the $\delta \mathcal{G}_{\alpha^{\prime} \text { corr. }}^{a_{1} a_{2} a_{3} a_{4}}$ contribution vanishes. Only at the lowest order in $\alpha^{\prime}$ this is true, since that contribution in $\mathcal{G}_{\alpha^{\prime} \text { corr. }}^{{ }^{a} a_{2} a_{2} a_{3} a_{4}}$ is only a constant, having a null variation. As soon as higher $\alpha^{\prime}$ contributions are taken into account, this is no longer valid since these contributions include the $A_{\mu}$ variations that arise from the covariant derivatives. Then it would be expected that, from $\alpha^{\prime 3}$ order on, new terms in the effective action would be needed to cancel these variations [24].

Our conclusion is that, using 4-point amplitudes it is not possible to construct and effective action which is has supersymmetric invariants at every order in $\alpha^{\prime}$, as was done in the abelian case [29].

\section{$2.4 \alpha^{\prime}$ expansion}

In this section we review the corresponding one of [16]. The $\alpha^{\prime}$ expansion of $f(s, t)$ in $(2.12)$ can be determined to any desired order in $\alpha^{\prime}$ using the Taylor expansion for $\ln \Gamma(1+z)^{8}$,

$$
\ln \Gamma(1+z)=-\gamma z+\sum_{k=2}^{\infty}(-1)^{k} \frac{\zeta(k)}{k} z^{k} \quad(-1<z \leq 1)
$$

as was remarked in [24]. In (2.20) $\gamma$ is the Euler-Mascheroni constant and $\zeta(k)$ is the Riemann Zeta function. This leads to:

$$
\alpha^{\prime 2} f(s, t)=\frac{1}{s t}\left[\exp \left\{\sum_{k=2}^{\infty} \frac{\zeta(k)}{k} \alpha^{\prime k}\left(s^{k}+t^{k}-(s+t)^{k}\right)\right\}-1\right] .
$$

In [16] it was seen that the first terms of the $\alpha^{\prime}$ series of this last expression could be rearranged as $^{9}$ :

$$
\begin{aligned}
\alpha^{\prime 2} f(s, t)= & -\frac{\pi^{2}}{6} \alpha^{\prime 2}+\zeta(3) u \alpha^{\prime 3}+\left[-\frac{\pi^{4}}{180}\left(s^{2}+t^{2}+u^{2}\right)+\frac{\pi^{4}}{120} s t\right] \alpha^{\prime 4} \\
& +\left[-\frac{\pi^{2}}{6} \zeta(3) s t u+\frac{1}{2} \zeta(5)\left(s^{2}+t^{2}+u^{2}\right) u\right] \alpha^{\prime 5} \\
+ & {\left[-\frac{\pi^{6}}{2880}\left(s^{2}+t^{2}+u^{2}\right)^{2}+\frac{\pi^{6}}{3024}\left(s^{2}+t^{2}+u^{2}\right)\left(s t+\frac{u^{2}}{2}\right)\right.} \\
& \left.+\left(\frac{\pi^{6}}{3024}+\frac{\zeta(3)^{2}}{2}\right)(s t u) u\right] \alpha^{\prime^{6}}+\mathcal{O}\left({\alpha^{\prime}}^{7}\right)
\end{aligned}
$$

where the relation (2.5) was taken into account.

At every order in $\alpha^{\prime}$, the coefficients of the series in (2.22) have been split into two types of terms, according to their appearance in the expansion of the abelian factor $\mathcal{G}$ in (2.2), after including the expansions of $f(t, u)$ and $f(u, s)$ : the first ones, which are symmetric in $(s, t, u)$ are the ones which will be present in $\mathcal{G}$, while the second ones will cancel, upon using condition (2.5). In the nonabelian factor $\mathcal{G}_{\alpha^{\prime} \text { corr. }}^{a_{1} a_{2} a_{3} a_{4}}$ the first type of terms will contribute as coefficients of $\operatorname{str}\left(\lambda^{a_{1}} \lambda^{a_{2}} \lambda^{a_{3}} \lambda^{a_{4}}\right)$, while

\footnotetext{
${ }^{8}$ See formula (10.44c) of [37], for example.

${ }^{9}$ The minus signs of the terms containing odd powers of $\zeta(3)$ in eq. (2.7) of [16] have been changed.
} 
the second ones will contribute as coefficients of $f$ - $f$ terms, in the following way:

$$
\begin{aligned}
& \alpha^{\prime 2} \mathcal{G}_{\alpha^{\prime} \text { corr. }}^{a_{1} a_{2} a_{3} a_{4}}(s, t, u)=\left\{-\pi^{2} \operatorname{str}\left(\lambda^{a_{1}} \lambda^{a_{2}} \lambda^{a_{3}} \lambda^{a_{4}}\right)\right\} \alpha^{\prime 2} \\
& +\left\{-\zeta(3)\left[f^{a_{1} a_{2} b} f_{b}^{a_{3} a_{4}} u+f^{a_{1} a_{3} b} f_{b}^{a_{2} a_{4}} s\right]\right\} \alpha^{\prime 3} \\
& +\left\{-\frac{\pi^{4}}{24}\left(s^{2}+t^{2}+u^{2}\right) \operatorname{str}\left(\lambda^{a_{1}} \lambda^{a_{2}} \lambda^{a_{3}} \lambda^{a_{4}}\right)+\frac{\pi^{4}}{360}\left[s(u-t) f^{a_{1} a_{2} b} f_{b}^{a_{3} a_{4}}\right.\right. \\
& \left.\left.+u(s-t) f^{a_{1} a_{3} b} f_{b}^{a_{2} a_{4}}+t(s-u) f^{a_{1} a_{4} b} f_{b}^{a_{2} a_{3}}\right]\right\} \alpha^{\prime 4} \\
& +\left\{-\pi^{2} \zeta(3) s t u \operatorname{str}\left(\lambda^{a_{1}} \lambda^{a_{2}} \lambda^{a_{3}} \lambda^{a_{4}}\right)\right. \\
& \left.-\frac{1}{2} \zeta(5)\left(s^{2}+t^{2}+u^{2}\right)\left(f^{a_{1} a_{2} b} f_{b}^{a_{3} a_{4}} u+f^{a_{1} a_{3} b} f_{b}^{a_{2} a_{4}} s\right)\right\} \alpha^{\prime 5} \\
& +\left\{-\frac{\pi^{6}}{480}\left(s^{2}+t^{2}+u^{2}\right)^{2} \operatorname{str}\left(\lambda^{a_{1}} \lambda^{a_{2}} \lambda^{a_{3}} \lambda^{a_{4}}\right)\right. \\
& +\frac{\pi^{6}}{6048}\left(s^{2}+t^{2}+u^{2}\right)\left[s(u-t) f^{a_{1} a_{2} b} f_{b}^{a_{3} a_{4}}\right. \\
& \left.+u(s-t) f^{a_{1} a_{3} b} f^{a_{2} a_{4}}+t(s-u) f^{a_{1} a_{4} b} f^{a_{2} a_{3}}{ }_{b}\right] \\
& \left.-\left(\frac{\pi^{6}}{3024}+\frac{\zeta(3)^{2}}{2}\right)(s t u)\left[f^{a_{1} a_{2} b} f_{b}^{a_{3} a_{4}} u+f^{a_{1} a_{3} b} f_{b}^{a_{2} a_{4}} s\right]\right\} \alpha^{\prime 6} \\
& +\mathcal{O}\left(\alpha^{\prime 7}\right) \text {. }
\end{aligned}
$$

In deriving the $f$ - $f$ terms of this expansion, the (2.5) condition together with the relations (A.13) and (A.14) were used.

\section{$2.5 \mathcal{O}\left(\alpha^{\prime 3}\right)$ and $\mathcal{O}\left(\alpha^{\prime 4}\right)$ tests}

The $\mathcal{O}\left(\alpha^{\prime 2}\right)$ test of formula (2.10) was already considered in subsection 2.2. In the present subsection we will make connection with higher $\alpha^{\prime}$ terms of the effective action which are sensible to 4-point amplitudes and which are already known in the literature.

Using integration by parts, equations of motion and the Jacobi identity (A.15), we have confirmed that the $\mathcal{O}\left(\alpha^{\prime 3}\right)$ terms of our effective action (2.10), obtained using the

$$
\alpha^{\prime 2} \mathcal{G}_{(1)}^{a_{1} a_{2} a_{3} a_{4}}(s, t, u)=-\zeta(3)\left[f^{a_{1} a_{2} b} f_{b}^{a_{3} a_{4}} u+f^{a_{1} a_{3} b} f_{b}^{a_{2} a_{4}} s\right] \alpha^{\prime 3}
$$

part of (2.23), match completely with the corresponding bosonic and fermionic terms of [16].

In the case of the $\mathcal{O}\left(\alpha^{\prime 4}\right)$ terms we have only considered the symmetrized ones, obtained from the

$$
\alpha^{\prime 2} \mathcal{G}_{(2)}^{a_{1} a_{2} a_{3} a_{4}}(s, t, u)=-\frac{\pi^{4}}{24}\left(s^{2}+t^{2}+u^{2}\right) \operatorname{str}\left(\lambda^{a_{1}} \lambda^{a_{2}} \lambda^{a_{3}} \lambda^{a_{4}}\right) \alpha^{\prime 4}
$$

part of (2.23). These terms are the ones which survive in the abelian limit. Besides using integration by parts and equations of motion, we have used the identities of Appendix A and we have arrived to the conclusion that only for the four fermion terms we have agreement with [16]. Although our four boson and two boson/two fermion terms disagree with the ones in [16], we have found some further agreement when comparing them with other existing versions in the literature, as we will comment in the next lines.

We have checked that the abelian limit of four boson terms agree with the $\partial^{4} F^{4}$ ones of [6]. We also expect our terms to agree with the symmetrized trace ones that would be obtained from $[23]^{10}$

\footnotetext{
${ }^{10}$ The complete $\mathcal{O}\left(\alpha^{\prime 4}\right)$ terms of [23], which also include the bosonic terms sensible to 5 and 6-point amplitudes, were recently confirmed in [38].
} 
since in this reference agreement was also found with [6] in the abelian limit.

In the case of the two boson/two fermion terms, we have checked that, in the abelian limit, they are equivalent to the corresponding ones in eq. (3.2) of [25], except for a global relative sign between the $\gamma^{\mu}$ and the $\gamma^{\mu \nu \rho}$ terms.

\subsection{Higher order terms in the effective action}

As an application of our nonabelian generalization (2.10) we now calculate the nonabelian $\mathcal{O}\left(\alpha^{15}\right)$ terms of the effective action which, as far as we know, have not been calculated before. Instead of using the symmetrized prescription for the Mandelstam variables (2.7), we now use

$$
s \rightarrow 2 D_{1} \cdot D_{2}, \quad t \rightarrow 2 D_{1} \cdot D_{4} \quad \text { and } \quad u \rightarrow 2 D_{1} \cdot D_{3} \quad .
$$

The terms of the effective action derived using this prescription will be on-shell equivalent to the ones in (2.10).

Using (2.26) for the $\mathcal{O}\left(\alpha^{\prime}\right)$ terms of $(2.23)$ in (2.10), we have

$$
\begin{aligned}
S_{(5)}^{\text {nonabel. }}= & 2 g^{2} \alpha^{\prime 5} \int d^{10} x \iiint \int\left\{\prod_{j=1}^{4} d^{10} x_{j} \delta^{(10)}\left(x-x_{j}\right)\right\} \\
\times & {\left[2 \pi^{2} \zeta(3) \operatorname{str}\left(\lambda^{a_{1}} \lambda^{a_{2}} \lambda^{a_{3}} \lambda^{a_{4}}\right)\left(D_{1} \cdot D_{2}\right)\left(D_{1} \cdot D_{4}\right)\left(D_{1} \cdot D_{3}\right)\right.} \\
& +\zeta(5)\left\{f^{a_{1} a_{2} b} f_{b}^{a_{3} a_{4}}\left(D_{1} \cdot D_{3}\right)+f^{a_{1} a_{3} b} f^{a_{2} a_{4}}{ }_{b}\left(D_{1} \cdot D_{2}\right)\right\} \\
& \left.\cdot\left\{\left(D_{1} \cdot D_{2}\right)^{2}+\left(D_{1} \cdot D_{4}\right)^{2}+\left(D_{1} \cdot D_{3}\right)^{2}\right\}\right] \\
\times & {\left[F_{a_{1} \mu \nu}\left(x_{1}\right) F_{a_{2}}^{\nu \rho}\left(x_{2}\right) F_{a_{3} \rho \sigma}\left(x_{3}\right) F_{a_{4}}^{\sigma \mu}\left(x_{4}\right)-\frac{1}{4} F_{a_{1} \mu \nu}\left(x_{1}\right) F_{a_{2}}^{\mu \nu}\left(x_{2}\right) F_{a_{3} \rho \sigma}\left(x_{3}\right) F_{a_{4}}^{\rho \sigma}\left(x_{4}\right)\right.} \\
+ & 2 i \bar{\psi}_{a_{1}}\left(x_{1}\right) \gamma^{\nu} D_{\rho} \psi\left(x_{2}\right)_{a_{2}} F_{a_{3} \nu \mu}\left(x_{3}\right) F_{a_{4}}^{\mu \rho}\left(x_{4}\right)-i \bar{\psi}_{a_{1}}\left(x_{1}\right) \gamma^{\mu \nu \rho} D^{\sigma} \psi\left(x_{2}\right)_{a_{2}} F_{a_{3} \mu \nu}\left(x_{3}\right) F_{a_{4} \rho \sigma}\left(x_{4}\right) \\
& \left.-\frac{1}{3} \bar{\psi}_{a_{1}}\left(x_{1}\right) \gamma_{\mu} D_{\nu} \psi\left(x_{2}\right)_{a_{2}} \bar{\psi}_{a_{3}}\left(x_{3}\right) \gamma^{\mu} D^{\nu} \psi\left(x_{4}\right)_{a_{4}}\right] .
\end{aligned}
$$

After performing the delta function integrations this leads to

$$
\begin{aligned}
S_{(5)}^{\text {nonabel. }}= & 4 \pi^{2} \zeta(3) g^{2} \alpha^{\prime 5} \int d^{10} x \operatorname{str}\left[D_{\lambda} D_{\tau} D_{\kappa} F_{\mu \nu} D^{\lambda} F^{\nu \rho} D^{\tau} F_{\rho \sigma} D^{\kappa} F^{\sigma \mu}\right. \\
& -\frac{1}{4} D_{\lambda} D_{\tau} D_{\kappa} F_{\mu \nu} D^{\lambda} F^{\mu \nu} D^{\tau} F_{\rho \sigma} D^{\kappa} F^{\rho \sigma}+2 i D_{\lambda} D_{\tau} D_{\kappa} \bar{\psi} \gamma^{\nu} D^{\lambda} D_{\rho} \psi D^{\tau} F_{\nu \mu} D^{\kappa} F^{\mu \rho} \\
& -i D_{\lambda} D_{\tau} D_{\kappa} \bar{\psi} \gamma^{\mu \nu \rho} D^{\lambda} D^{\sigma} \psi D^{\tau} F_{\mu \nu} D^{\kappa} F_{\rho \sigma} \\
& \left.-\frac{1}{3} D_{\lambda} D_{\tau} D_{\kappa} \bar{\psi} \gamma_{\mu} D^{\lambda} D_{\nu} \psi D^{\tau} \bar{\psi} \gamma^{\mu} D^{\kappa} D^{\nu}\right] \\
+ & 2 \zeta(5) g^{2} \alpha^{\prime 5} \mathcal{S}_{(5), f-f}
\end{aligned}
$$

where $\mathcal{S}_{(5), f-f}$ represents the term with structure constants, written explicitly in Appendix C. The abelian bosonic terms of (2.28) match completely with the corresponding ones of $[29]^{11}$. These terms were compared in [29] with the six-derivative ones of [13], finding agreement, up to field redefinitions.

A feature that was not present in the previous $\mathcal{O}\left(\alpha^{\prime k}\right)$ terms (with $k=2,3,4$ ), and that appears in (2.28) and in all higher order $\alpha^{\prime}$ terms, is the fact that, for the same $k$-th order in $\alpha^{\prime}$, besides $\zeta(k)$ multiplying a supersymmetric invariant, other global coefficients are present multiplying other

\footnotetext{
${ }^{11}$ Except for a minus sign that we have taken into account in our formula 2.28 .
} 
supersymmetric invariants. These coefficients will contain, as part of them, products of $\zeta(n)$, where $n$ is odd at least once (as, for example, the $\pi^{2} \zeta(3) \sim \zeta(2) \zeta(3)$ coefficient in (2.28)). So, it is expected that the ratio between these different global coefficients is not a fractional number. This fact has been used in [24, 25, 29], among other things, to study the independent supersymmetric invariants that arise in the effective action, when considering the Noether method.

It is straight forward to construct the explicit expression of the $\mathcal{O}\left(\alpha^{\prime 6}\right)$ (or higher order) terms of the effective action, using the series in (2.23).

\section{Closed superstring 4-point effective action}

\subsection{The general formula}

Let us review some basic facts about graviton scattering amplitudes in type II superstrings [39]. The tree level amplitude of four gravitons is given by [17]

$$
\mathcal{A}_{\text {grav. }}^{(4)}=16 i \kappa^{2} e^{2 \Phi} G(s, t, u) K_{\text {grav. }}^{(4)},
$$

where

$$
G(s, t, u)=\frac{1}{s t u} \frac{\Gamma\left(1-\frac{1}{4} \alpha^{\prime} s\right) \Gamma\left(1-\frac{1}{4} \alpha^{\prime} t\right) \Gamma\left(1-\frac{1}{4} \alpha^{\prime} u\right)}{\Gamma\left(1+\frac{1}{4} \alpha^{\prime} s\right) \Gamma\left(1+\frac{1}{4} \alpha^{\prime} s\right) \Gamma\left(1+\frac{1}{4} \alpha^{\prime} s\right)} .
$$

In (3.1) $\kappa$ is the ten-dimensional gravitational coupling constant, $s, t, u$ are the same Mandelstam variables of (2.4) and the kinematical factor $K_{\text {grav }}^{(4)}$, which is of eighth order in momenta, is given by

$$
K_{\text {grav. }}^{(4)}=t_{(8)}^{\mu_{1} \nu_{1} \mu_{2} \nu_{2} \mu_{3} \nu_{3} \mu_{4} \nu_{4}} t_{(8)}^{\lambda_{1} \rho_{1} \lambda_{2} \rho_{2} \lambda_{3} \rho_{3} \lambda_{4} \rho_{4}} \prod_{j=1}^{4} \zeta_{j \mu_{j} \lambda_{j}} k_{j \nu_{j}} k_{j \rho_{j}}
$$

In (3.3), the $t_{(8)}$ tensor is exactly the same one appearing in the four gluon scattering amplitude (B.4) and $\zeta_{\mu \nu}=\zeta_{\nu \mu}$ is the graviton polarization tensor.

We will see in the next subsection that the $\alpha^{\prime}$ expansion of the Gamma factor in (3.1) contains poles only in the leading term, in analogy to the corresponding Gamma factor in the open superstring amplitude (2.13). So, besides the leading contribution to the scattering amplitude, the rest of the $\alpha^{\prime}$ terms contribute as 1-particle irreducible diagrams only, being possible to apply the same technique of $[29]$.

It is very well known, that the first non zero $\alpha^{\prime}$ contribution in the effective action arises at order $\alpha^{\prime 3}$ with the characteristic coefficient $\zeta(3)$ and $R^{4}$ type of terms [4]. In particular this means that, besides the leading $\mathrm{D}=10$ Einstein-Hilbert term, there are no $\alpha^{\prime} R^{2}$ nor $\alpha^{\prime 2} R^{3}$ corrections in the effective action. The $\alpha^{\prime 3} R^{4}$ term in the action which is consistent with the $\alpha^{\prime 3}$ terms of (3.1) has the form $[4,39]$

$$
\frac{\zeta(3) \alpha^{\prime 3}}{2^{9} \cdot 4 ! \kappa^{2}} \int d^{10} x \sqrt{-g} e^{-2 \Phi} t_{(8)}^{\mu_{1} \nu_{1} \mu_{2} \nu_{2} \mu_{3} \nu_{3} \mu_{4} \nu_{4}} t_{(8)}^{\lambda_{1} \rho_{1} \lambda_{2} \rho_{2} \lambda_{3} \rho_{3} \lambda_{4} \rho_{4}} R_{\mu_{1} \nu_{1} \lambda_{1} \rho_{1}} R_{\mu_{2} \nu_{2} \lambda_{2} \rho_{2}} R_{\mu_{3} \nu_{3} \lambda_{3} \rho_{3}} R_{\mu_{4} \nu_{4} \lambda_{4} \rho_{4}} .
$$


So, our proposal for the graviton 4-point functions in the effective action is the following:

$$
\begin{aligned}
& S_{\text {eff }}^{\text {grav. }}=\frac{1}{2 \kappa^{2}} \int d^{10} x \sqrt{-g} R \\
& +\frac{1}{2^{4} \cdot 4 ! \kappa^{2}} \int d^{10} x \sqrt{-g} \int\left\{\prod_{j=1}^{4} d^{10} x_{j} \delta^{(10)}\left(x-x_{j}\right)\right\} \hat{G}\left(2 \nabla_{1} \cdot \nabla_{2}, 2 \nabla_{1} \cdot \nabla_{4}, 2 \nabla_{1} \cdot \nabla_{3}\right) \\
& e^{-2 \Phi} t_{(8)}^{\mu_{1} \nu_{1} \mu_{2} \nu_{2} \mu_{3} \nu_{3} \mu_{4} \nu_{4}} t_{(8)}^{\lambda_{1} \rho_{1} \lambda_{2} \rho_{2} \lambda_{3} \rho_{3} \lambda_{4} \rho_{4}} R_{\mu_{1} \nu_{1} \lambda_{1} \rho_{1}}\left(x_{1}\right) R_{\mu_{2} \nu_{2} \lambda_{2} \rho_{2}}\left(x_{2}\right) R_{\mu_{3} \nu_{3} \lambda_{3} \rho_{3}}\left(x_{3}\right) R_{\mu_{4} \nu_{4} \lambda_{4} \rho_{4}}\left(x_{4}\right) .
\end{aligned}
$$

In (3.5) the $\hat{G}$ operator is defined in terms of $G$ by means of

$$
\hat{G}(s, t, u)=G(s, t, u)-\frac{1}{s t u},
$$

in analogy to (2.12). In the next subsection it will become clear that the $\alpha^{\prime 3}$ terms of (3.5) match exactly with (3.4). Note that, as there is no need to prove any supersymmetry ${ }^{12}$, the $\hat{G}$ operator does not need to be evaluated in a symmetrized way, as was done in (2.1) [29]. So, when using the prescription (2.6), the Mandelstam variables have just been introduced as in (2.4).

In analogy to the open superstring case (2.8), (2.9) and (2.10), in (3.5) we have explicitly separated the leading contribution, given by the $\mathrm{D}=10$ Einstein-Hilbert term, from the rest of the $\alpha^{\prime}$ correction terms.

Strictly speaking, we would have to verify that the four graviton scattering amplitude derived from (3.5) is exactly expression (3.1), as was done in [29], and as we did in Appendix B for nonabelian open superstring. We have omitted this proof since the procedure we would have to follow is directly the same one.

Now, what remains is to include the rest of the NS-NS fields, namely, the Kalb-Ramond field $B_{\mu \nu}$ and the dilaton $\Phi$. The amplitude involving these states can be calculated as before just noting that the dilaton polarization is a scalar and that the corresponding one for the $B_{\mu \nu}$ is an antisymmetric tensor, $\zeta_{\mu \nu}=-\zeta_{\nu \mu}$. In order to derive the effective action for these fields, we would have to reconsider the scattering amplitude approach for their $\alpha^{3}$ terms, as was done for the graviton. Fortunately, this has been done time ago [33]. It was shown in [33] that this term is given exactly by (3.4) by replacing the curvature tensor by the combination

$$
\bar{R}_{\mu \nu}^{\lambda \rho}=R_{\mu \nu}^{\lambda \rho}+e^{-\Phi} \nabla_{[\mu} H_{\nu]}^{\lambda \rho}-\delta_{[\mu}^{[\lambda} \nabla_{\nu]} \nabla^{\rho]} \Phi
$$

where $H_{\mu \nu \lambda}=\partial_{[\mu} B_{\nu \lambda]}$. Therefore the effective action for all the massless NS-NS states is given by (3.8) by changing $R$ by $\bar{R}$. So our final expression for the effective action NS-NS terms, sensible to 4 -point amplitudes, is the following:

$$
\begin{aligned}
& S_{e f f}^{\mathrm{NS}-\mathrm{NS}}=\frac{1}{2 \kappa^{2}} \int d^{10} x \sqrt{-g}\left(R-\frac{1}{12} e^{-\Phi} H_{\mu \nu \lambda} H^{\mu \nu \lambda}-\frac{1}{2} \partial_{\mu} \Phi \partial^{\mu} \Phi\right) \\
& +\frac{1}{2^{4} \cdot 4 ! \kappa^{2}} \int d^{10} x \sqrt{-g} \int\left\{\prod_{j=1}^{4} d^{10} x_{j} \delta^{(10)}\left(x-x_{j}\right)\right\} \hat{G}\left(2 \nabla_{1} \cdot \nabla_{2}, 2 \nabla_{1} \cdot \nabla_{4}, 2 \nabla_{1} \cdot \nabla_{3}\right) \\
& e^{-2 \Phi} t_{(8)}^{\mu_{1} \nu_{1} \mu_{2} \nu_{2} \mu_{3} \nu_{3} \mu_{4} \nu_{4}} t_{(8)}^{\lambda_{1} \rho_{1} \lambda_{2} \rho_{2} \lambda_{3} \rho_{3} \lambda_{4} \rho_{4}} \bar{R}_{\mu_{1} \nu_{1} \lambda_{1} \rho_{1}}\left(x_{1}\right) \bar{R}_{\mu_{2} \nu_{2} \lambda_{2} \rho_{2}}\left(x_{2}\right) \bar{R}_{\mu_{3} \nu_{3} \lambda_{3} \rho_{3}}\left(x_{3}\right) \bar{R}_{\mu_{4} \nu_{4} \lambda_{4} \rho_{4}}\left(x_{4}\right)
\end{aligned}
$$

\footnotetext{
${ }^{12}$ The complete action is indeed supersymmetric but, at this level, we are just dealing with the NS-NS sector which is completely bosonic.
} 


\subsection{Higher order terms in the effective action}

We will begin writing and explicit $\alpha^{\prime}$ series for $\hat{G}(s, t, u)$, in analogy to what we did with $f(s, t)$ in (2.22). For this purpose we first notice that $G(s, t, u)$ in $(3.2)$ contains three factors of the form $\Gamma(1-z) / \Gamma(1+z)$, that may be expanded using the Taylor series (2.20), giving

$$
\ln \frac{\Gamma(1-z)}{\Gamma(1+z)}=2 \gamma z+2 \sum_{n=1}^{\infty} \frac{\zeta(2 n+1)}{2 n+1} z^{2 n+1} \quad(|z|<1) .
$$

Using this result and condition (2.5), we then have that

$$
\hat{G}(s, t, u)=\frac{1}{s t u}\left[\exp \left\{2 \sum_{k=1}^{\infty}\left(\frac{\alpha^{\prime}}{4}\right)^{2 k+1} \frac{\zeta(2 k+1)}{2 k+1}\left(s^{2 k+1}+t^{2 k+1}+u^{2 k+1}\right)\right\}-1\right] .
$$

This expression shows that the coefficients of the $\alpha^{\prime}$ series of $\hat{G}(s, t, u)$ are all given in terms of odd valued $\zeta(n)$. Besides this, it may be proved that $\left(s^{2 k+1}+t^{2 k+1}+u^{2 k+1}\right)$ is always divisible by $s t u$, when condition (2.5) is taken into account, so the series in (3.10) has no poles. In fact, writing only the first terms of the series, we have:

$$
\begin{aligned}
\hat{G}(s, t, u)= & 2 \zeta(3)\left(\frac{\alpha^{\prime}}{4}\right)^{3}+2 \zeta(5)\left(s^{2}+s t+t^{2}\right)\left(\frac{\alpha^{\prime}}{4}\right)^{5}+2 \zeta(3)^{2} s t u\left(\frac{\alpha^{\prime}}{4}\right)^{6} \\
& +2 \zeta(7)\left(s^{4}+2 t s^{3}+3 t^{2} s^{2}+2 t^{3} s+t^{4}\right)\left(\frac{\alpha^{\prime}}{4}\right)^{7} \\
& +4 \zeta(3) \zeta(5) s t u\left(s^{2}+s t+t^{2}\right)\left(\frac{\alpha^{\prime}}{4}\right)^{8}+\mathcal{O}\left(\alpha^{\prime 9}\right) .
\end{aligned}
$$

Let us write the first terms of the expansion in gravitational sector only. The first term of the expansion is of order $\left(\alpha^{\prime}\right)^{3}$ and it is given by (3.4) multiplied $\zeta(3)$. Note that there is no term of order $\left(\alpha^{\prime}\right)^{4}[34]$. So the first new correction is at $\left(\alpha^{\prime}\right)^{5}$ and it is give by ${ }^{13}$

$$
\begin{aligned}
& \frac{\zeta(5) \alpha^{\prime 5}}{2^{11} \cdot 4 ! \kappa^{2}} \int d^{10} x \sqrt{-g} t_{(8)}^{\mu_{1} \nu_{1} \mu_{2} \nu_{2} \mu_{3} \nu_{3} \mu_{4} \nu_{4}} t_{(8)}^{\lambda_{1} \rho_{1} \lambda_{2} \rho_{2} \lambda_{3} \rho_{3} \lambda_{4} \rho_{4}} \\
& \quad\left[2 \nabla_{\sigma} \nabla_{\tau} R_{\mu_{1} \nu_{1} \lambda_{1} \rho_{1}} \nabla^{\sigma} \nabla^{\tau} R_{\mu_{2} \nu_{2} \lambda_{2} \rho_{2}} R_{\mu_{3} \nu_{3} \lambda_{3} \rho_{3}} R_{\mu_{4} \nu_{4} \lambda_{4} \rho_{4}}\right. \\
& \left.\quad+\nabla_{\sigma} \nabla_{\tau} R_{\mu_{1} \nu_{1} \lambda_{1} \rho_{1}} \nabla^{\sigma} R_{\mu_{2} \nu_{2} \lambda_{2} \rho_{2}} \nabla^{\tau} R_{\mu_{3} \nu_{3} \lambda_{3} \rho_{3}} R_{\mu_{4} \nu_{4} \lambda_{4} \rho_{4}}\right] .
\end{aligned}
$$

The next correction is at $\left(\alpha^{\prime}\right)^{6}$ and it is given by

$$
\begin{aligned}
& \frac{\zeta(3)^{2} \alpha^{\prime 6}}{2^{12} \cdot 4 ! \kappa^{2}} \int d^{10} x \sqrt{-g} t_{(8)}^{\mu_{1} \nu_{1} \mu_{2} \nu_{2} \mu_{3} \nu_{3} \mu_{4} \nu_{4}} t_{(8)}^{\lambda_{1} \rho_{1} \lambda_{2} \rho_{2} \lambda_{3} \rho_{3} \lambda_{4} \rho_{4}} \\
& {\left[\nabla_{\sigma} \nabla_{\tau} \nabla_{\omega} R_{\mu_{1} \nu_{1} \lambda_{1} \rho_{1}} \nabla^{\sigma} R_{\mu_{2} \nu_{2} \lambda_{2} \rho_{2}} \nabla^{\tau} R_{\mu_{3} \nu_{3} \lambda_{3} \rho_{3}} \nabla^{\omega} R_{\mu_{4} \nu_{4} \lambda_{4} \rho_{4}}\right] .}
\end{aligned}
$$

Finally we write the order $\left(\alpha^{\prime}\right)^{7}$

$$
\begin{gathered}
\frac{\zeta(7) \alpha^{\prime 7}}{2^{13} \cdot 4 ! \kappa^{2}} \int d^{10} x \sqrt{-g} t_{(8)}^{\mu_{1} \nu_{1} \mu_{2} \nu_{2} \mu_{3} \nu_{3} \mu_{4} \nu_{4}} t_{(8)}^{\lambda_{1} \rho_{1} \lambda_{2} \rho_{2} \lambda_{3} \rho_{3} \lambda_{4} \rho_{4}} \\
{\left[2 \nabla_{\sigma} \nabla_{\tau} \nabla_{\omega} \nabla_{\kappa} R_{\mu_{1} \nu_{1} \lambda_{1} \rho_{1}} \nabla^{\sigma} \nabla^{\tau} \nabla^{\omega} \nabla^{\kappa} R_{\mu_{2} \nu_{2} \lambda_{2} \rho_{2}} R_{\mu_{3} \nu_{3} \lambda_{3} \rho_{3}} R_{\mu_{4} \nu_{4} \lambda_{4} \rho_{4}}\right.} \\
+4 \nabla_{\sigma} \nabla_{\tau} \nabla_{\omega} \nabla_{\kappa} R_{\mu_{1} \nu_{1} \lambda_{1} \rho_{1}} \nabla^{\sigma} \nabla^{\tau} \nabla^{\omega} R_{\mu_{2} \nu_{2} \lambda_{2} \rho_{2}} \nabla^{\kappa} R_{\mu_{3} \nu_{3} \lambda_{3} \rho_{3}} R_{\mu_{4} \nu_{4} \lambda_{4} \rho_{4}} \\
\left.+3 \nabla_{\sigma} \nabla_{\tau} \nabla_{\kappa} R_{\mu_{1} \nu_{1} \lambda_{1} \rho_{1}} \nabla^{\sigma} \nabla^{\tau} R_{\mu_{2} \nu_{2} \lambda_{2} \rho_{2}} \nabla^{\omega} \nabla^{\kappa} R_{\mu_{3} \nu_{3} \lambda_{3} \rho_{3}} R_{\mu_{4} \nu_{4} \lambda_{4} \rho_{4}}\right] .
\end{gathered}
$$

\footnotetext{
${ }^{13}$ We consider gravitational excitations only. Therefore, we use the combination $\left(\kappa e^{\Phi}\right)$ as the gravitational constant $\kappa$ in the subsequent formulae.
} 
It would be interesting to determine the supersymmetric extension of (3.8). In order to do this, it is necessary to compute scattering amplitudes involving up to 16 fermions. It will be also necessary to include Ramond-Ramond fields. It is much more convenient to use the constraints imposed by the corresponding supersymmetry by following [40] (recall there are two possible supersymmetries for the same NS-NS massless fields, type IIA and type IIB).

The calculation we have done is at tree-level for the string. It is known that the term of the effective action $\zeta(3) R^{4}$ receives corrections beyond one-loop and even non-perturbative ones [41]. Namely, for both type II superstrings, this term receives corrections at tree and at one-loop level in string perturbation theory. For the type IIB superstring, this term also receives non-perturbative corrections. It is also known that the term $\zeta(5) \nabla^{4} R^{4}$ receives perturbative corrections at tree-level, as we see in (3.12), and at two-loops and non-perturbative ones for the type IIB superstring [36]. There has been investigations to determine the perturbative and non-perturbative structure of some higher derivative terms [35]. It would be interesting to investigate if the remaining terms in (3.8) have similar properties ${ }^{14}$.

\section{Final remarks and conclusions}

We have derived two new results for effective actions in superstring theory. Both of them consist in explicit expressions for the infinite $\alpha^{\prime}$ series and they are based in a recent derivation of the effective action for the 4-point functions in abelian open superstring theory [29].

Our first result consists in the nonabelian generalization of [29]. We have tested our nonabelian action (2.10), at $\alpha^{3}$ and $\alpha^{\prime 4}$ order, with the corresponding expressions calculated by other methods $[6,23,16,25]$, which are different to the scattering amplitude approach. In most of the cases we have found agreement with the previous results.

Our second result consists in the effective action for the 4-point functions in the NS-NS sector of closed superstring theory. In the context of closed superstring effective actions, we have reproduced the known results for terms at $\mathcal{O}\left(\alpha^{\prime 3}\right)[4,33]$ and explicitly found the tensor structure of some higher derivative terms discussed in $[35,36]$. Our action (3.8) also confirms the non existence of $\mathcal{O}\left(\alpha^{\prime 4}\right)$ terms which are sensible to (tree level) 4-point amplitudes [34].

We would like to stress that the only Ansatz needed to compute our on-shell effective actions, sensible to 4-point amplitudes, at any desired order in $\alpha^{\prime}$, is the one done in (2.10) and (3.8). This should be contrasted with the previous traditional method of going, order by order in $\alpha^{\prime}$, proposing the most general structure of the four field terms, with coefficients to be determined by consistency with superstring scattering amplitudes (see $[14,16]$, for example).

We have used our effective actions (2.10) and (3.8) to derive higher order $\alpha^{\prime}$ corrections, as an application. These $\mathcal{O}\left(\alpha^{\prime k}\right)$ terms are by no means a complete list, in the sense that other terms which are sensible to higher n-point scattering amplitudes $(n>4)$ should also be present at the same $\alpha^{\prime k}$ order.

As a final remark we would like to comment on the $\zeta(n)$ dependence in the $\alpha^{\prime}$ series of the Gamma factors which appear in the 4-point amplitudes (2.13) and (3.1). It was used in [24] that the Taylor series for $\ln \Gamma(1+z)$, eq. (2.20), could make manifest the $\zeta(n)$ dependence of the Gamma factor for the open superstring (see eq. (2.21)). It is clear then, except for the zero $\alpha^{\prime}$ order and the low order null coefficients, that the rest of the coefficients of the $\alpha^{\prime}$ series are all composed of $\zeta(n)$ factors [24, 29]. The analog result for the closed superstring Gamma factor is given in (3.11) and it is quite remarkable that in this case only odd valued $\zeta(n)$ factors appear as coefficients of the $\alpha^{\prime}$ series.

\footnotetext{
${ }^{14}$ We thank to Pierre Vanhove for useful comments about these properties.
} 


\section{Acknowledgements}

R. M. would like to thank N. Berkovits and F. Brandt for useful conversations and the Brazilian agencies CNPq and FAPEMIG for partial financial support in this research. O. C. would like to thank J. Drummond and P. Vanhove for useful comments, FONDECYT grant 3000026 for partial financial support and the Instituto Nazionale de Fisica Nucleare for a postdoctoral fellowship.

\section{A. Conventions and Identities}

1. Metric:

$$
\eta_{\mu \nu}=\operatorname{diag}(-,+, \ldots,+)
$$

2. Spacetime indexes:

$$
\begin{aligned}
\alpha, \beta & \rightarrow \text { spinor components } \\
\kappa, \lambda, \mu, \nu, \rho, \sigma, \tau, \phi, \omega & \rightarrow \text { vector components }
\end{aligned}
$$

3. Spinors and Dirac Matrices:

Through out this paper all spinors are 10-dimensional Majorana-Weyl, with 16 real components.

The Dirac $\gamma^{\mu}$ matrices satisfy the Clifford algebra

$$
\left\{\gamma^{\mu}, \gamma^{\nu}\right\}=-2 \eta^{\mu \nu}
$$

Antisymmetric products of $p \gamma^{\mu}$ matrices, $\gamma_{\mu_{1} \mu_{2} \ldots \mu_{p}}$, are defined with a global factor $1 / p$ !. For $\gamma_{\mu \nu \rho}$ we have the following identities:

$$
\begin{gathered}
\gamma_{\mu \nu \rho}=\gamma_{\mu} \gamma_{\nu} \gamma_{\rho}+\gamma_{\mu} \eta_{\nu \rho}-\gamma_{\nu} \eta_{\rho \mu}+\gamma_{\rho} \eta_{\mu \nu} \\
\gamma_{\mu \nu \rho}=\gamma_{\mu \nu} \gamma_{\rho}+\gamma_{\mu} \eta_{\nu \rho}-\gamma_{\nu} \eta_{\rho \mu}
\end{gathered}
$$

A useful identity for Majorana-Weyl spinors:

$$
\bar{\chi}_{1} \gamma_{\mu_{1} \ldots \mu_{p}} \chi_{2}= \pm(-1)^{p(p+1) / 2} \bar{\chi}_{2} \gamma_{\mu_{1} \ldots \mu_{p}} \chi_{1},
$$

where the additional plus (minus) sign, before the $(-1)^{p(p+1) / 2}$ factor, is used for anticommuting (commuting) spinors.

A Fierz identity:

$$
\bar{\chi}_{1} \gamma_{\mu} \chi_{2} \bar{\chi}_{3} \gamma_{\mu} \chi_{4}+\bar{\chi}_{1} \gamma_{\mu} \chi_{3} \bar{\chi}_{4} \gamma_{\mu} \chi_{2}+\bar{\chi}_{1} \gamma_{\mu} \chi_{4} \bar{\chi}_{2} \gamma_{\mu} \chi_{3}=0
$$

In the way in which (A.6) has been written ,it is valid for all Majorana-Weyl spinors $\chi_{j}$ being commuting as well as all of them being anticommuting.

4. Gauge group, generators and structure constants:

Gauge fields are matrices in the $U(N)$ internal space, so that $A_{\mu}=A_{a}^{\mu} \lambda^{a}$, where the hermitian generators $\lambda^{a}$ are in the adjoint representation

$$
\left(\lambda^{a}\right)^{b c}=-i f^{a b c}
$$


and they satisfy the usual relations

$$
\left[\lambda^{a}, \lambda^{b}\right]=i f_{c}^{a b} \lambda^{c}, \quad \operatorname{tr}\left(\lambda^{a} \lambda^{b}\right)=\delta^{a b},
$$

for real structure constants $f^{a b c}$.

The field strength and the covariant derivative are defined by

$$
\begin{aligned}
F_{\mu \nu} & =\partial_{\mu} A_{\nu}-\partial_{\nu} A_{\mu}-i g\left[A_{\mu}, A_{\nu}\right], \\
D_{\mu} \phi & =\partial_{\mu} \phi-i g\left[A_{\mu}, \phi\right],
\end{aligned}
$$

and they are related by the identity

$$
\left[D_{\mu}, D_{\nu}\right] \phi=-i g\left[F_{\mu \nu}, \phi\right] .
$$

Covariant derivatives of field strengths satisfy the Bianchi identity:

$$
D^{\mu} F^{\nu \rho}+D^{\rho} F^{\mu \nu}+D^{\nu} F^{\rho \mu}=0 .
$$

The following expressions naturally appear in the scattering amplitude (2.13):

$$
\begin{aligned}
& T_{1}=\operatorname{tr}\left(\lambda^{a_{1}} \lambda^{a_{2}} \lambda^{a_{3}} \lambda^{a_{4}}\right)+\operatorname{tr}\left(\lambda^{a_{4}} \lambda^{a_{3}} \lambda^{a_{2}} \lambda^{a_{1}}\right), \\
& T_{2}=\operatorname{tr}\left(\lambda^{a_{1}} \lambda^{a_{3}} \lambda^{a_{2}} \lambda^{a_{4}}\right)+\operatorname{tr}\left(\lambda^{a_{4}} \lambda^{a_{2}} \lambda^{a_{3}} \lambda^{a_{1}}\right), \\
& T_{3}=\operatorname{tr}\left(\lambda^{a_{1}} \lambda^{a_{2}} \lambda^{a_{4}} \lambda^{a_{3}}\right)+\operatorname{tr}\left(\lambda^{a_{3}} \lambda^{a_{4}} \lambda^{a_{2}} \lambda^{a_{1}}\right) .
\end{aligned}
$$

For them

$$
T_{2}-T_{1}=-f^{a_{1} a_{4} b} f_{b}^{a_{2} a_{3}}, \quad T_{3}-T_{2}=+f^{a_{1} a_{3} b} f_{b}^{a_{2} a_{4}}, \quad T_{1}-T_{3}=-f^{a_{1} a_{2} b} f^{a_{3} a_{4}} .
$$

Summing all three relations comes the Jacobi identity:

$$
f^{a_{1} a_{4} b} f_{b}^{a_{2} a_{3}}-f^{a_{1} a_{3} b} f_{b}^{a_{2} a_{4}}+f^{a_{1} a_{2} b} f_{b}^{a_{3} a_{4}}=0 .
$$

5. Identity for the $t_{(8)}$ tensor:

The $t_{(8)}$ tensor $^{15}$, characteristic of the 4 boson scattering amplitude, is antisymmetric on each pair $\mu_{j} \nu_{j}(j=1,2,3,4)$ and is symmetric under any exchange of such of pairs. It allows to write the identity

$$
\begin{aligned}
P(1,2,3,4) \quad t_{\mu_{1} \nu_{1} \mu_{2} \nu_{2} \mu_{3} \nu_{3} \mu_{4} \nu_{4}}^{(8)} A_{1}^{\mu_{1} \nu_{1}} A_{2}^{\mu_{2} \nu_{2}} A_{3}^{\mu_{3} \nu_{3}} A_{4}^{\mu_{4} \nu_{4}}= \\
P(1,2,3,4)\left(A_{1 \mu \nu} A_{2}^{\nu \rho} A_{3 \rho \sigma} A_{4}^{\sigma \mu}-\frac{1}{4} A_{1 \mu \nu} A_{2}^{\mu \nu} A_{3 \rho \sigma} A_{4}^{\rho \sigma}\right),
\end{aligned}
$$

for any symmetric operator $P(1,2,3,4)$, that acts on index $j$ of an antisymmetric tensor $A_{j}^{\mu \nu}$ $(j=1,2,3,4)$.

\section{B. Derivation of the 4-particle scattering amplitudes from the effective action}

In this Appendix we follow closely the steps done for photons in the corresponding Appendix of [29]. But before going into the details, we comment on the 4-particle scattering processes that we are

\footnotetext{
${ }^{15} \mathrm{An}$ explicit expression for it may be found in equation (4.A.21) of [17].
} 


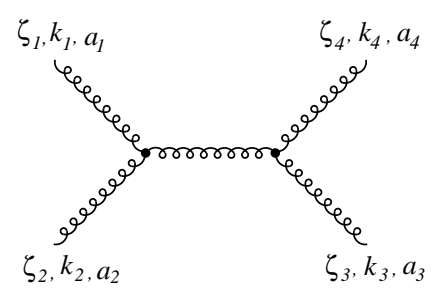

(a)

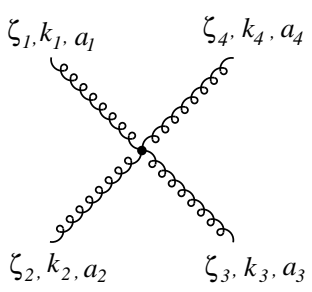

(b)

Figure 1: Type of tree diagrams which contribute to the 4-boson scattering amplitude in Super Yang-Mills theory. The diagram in (a) is 1-particle reducible, while the diagram in (b) is 1-particle irreducible.

considering (at tree level). It happens that they always present 1-particle reducible and irreducible diagrams, as the ones shown in (a), in figure 1. The 1-particle reducible diagrams arise only in the zero order $\alpha^{\prime}$ contribution of the effective theory, that is, in the Super Yang-Mills term $\left(S_{\mathrm{SYM}}\right)$.

Since the method followed to derive the abelian formula (2.1) is based in the effective action as the generator of 1-particle irreducible diagrams only, we have excluded the zero order $\alpha^{\prime}$ contribution in (2.12) and (2.10) and treated it separately in the $S_{\mathrm{SYM}}$ action of (2.8). So, when deriving the 4-point scattering amplitudes in section B.3 of this Appendix, we will calculate them as

$$
\mathcal{A}^{(4)}=\mathcal{A}_{(0)}^{(4)}+\mathcal{A}_{\alpha^{\prime} \text { corr. }}^{(4)}
$$

where the zero order and the $\alpha^{\prime}$ contribution, $\mathcal{A}_{(0)}^{(4)}$ and $\mathcal{A}_{\alpha^{\prime} \text { corr. }}^{(4)}$, will be calculated from the $S_{\mathrm{SYM}}$ and $S_{\alpha^{\prime} \text { corr. }}^{\text {nonabel. }}$ actions, respectively.

\section{B.1 4-particle scattering amplitudes in SYM}

The (on-shell) tree level 4-point amplitudes in Super Yang-Mills theory can be directly obtained from the $\alpha^{\prime} \rightarrow 0$ limit of the corresponding amplitudes in Open Superstring theory. They are all given by the common expression

$$
\mathcal{A}_{(0)}^{(4)}=8 i g^{2}(2 \pi)^{10} \delta^{(10)}\left(k_{1}+k_{2}+k_{3}+k_{4}\right) f_{(0)}^{a_{1} a_{2} a_{3} a_{4}}\left(k_{1}, k_{2}, k_{3}, k_{4}\right) K^{(4)}
$$

where

$$
\begin{aligned}
f_{(0)}^{a_{1} a_{2} a_{3} a_{4}}\left(k_{1}, k_{2}, k_{3}, k_{4}\right)= & \left\{\operatorname{tr}\left(\lambda^{a_{1}} \lambda^{a_{2}} \lambda^{a_{3}} \lambda^{a_{4}}\right)+\operatorname{tr}\left(\lambda^{a_{4}} \lambda^{a_{3}} \lambda^{a_{2}} \lambda^{a_{1}}\right)\right\} \cdot \frac{1}{s t} \\
& +\left\{\operatorname{tr}\left(\lambda^{a_{1}} \lambda^{a_{3}} \lambda^{a_{2}} \lambda^{a_{4}}\right)+\operatorname{tr}\left(\lambda^{a_{4}} \lambda^{a_{2}} \lambda^{a_{3}} \lambda^{a_{1}}\right)\right\} \cdot \frac{1}{t u} \\
& +\left\{\operatorname{tr}\left(\lambda^{a_{1}} \lambda^{a_{2}} \lambda^{a_{4}} \lambda^{a_{3}}\right)+\operatorname{tr}\left(\lambda^{a_{3}} \lambda^{a_{4}} \lambda^{a_{2}} \lambda^{a_{1}}\right)\right\} \cdot \frac{1}{u s},
\end{aligned}
$$

and where $K^{(4)}$ denotes the kinematic factor corresponding to each scattering process:

1. 4 bosons:

$$
K_{4 \mathrm{~b}}=t_{(8)}^{\mu_{1} \nu_{1} \mu_{2} \nu_{2} \mu_{3} \nu_{3} \mu_{4} \nu_{4}} \zeta_{\mu_{1}}^{1} k_{\nu_{1}}^{1} \zeta_{\mu_{2}}^{2} k_{\nu_{2}}^{2} \zeta_{\mu_{3}}^{3} k_{\nu_{3}}^{3} \zeta_{\mu_{4}}^{4} k_{\nu_{4}}^{4}
$$

where $\zeta_{j}$ and $k_{j}$ denote the $j$-th boson polarization and momentum, respectively.

$t_{(8)}^{\mu_{1} \nu_{1} \mu_{2} \nu_{2} \mu_{3} \nu_{3} \mu_{4} \nu_{4}}$ is a completely known tensor which satisfies, for example, the identity (A.16). 
2. 2 bosons and 2 fermions:

$$
\begin{aligned}
K_{2 \mathrm{~b} / 2 \mathrm{f}}=\frac{1}{8}[ & \left(\bar{u}_{1} \zeta_{3} u_{2}\right)\left\{\left(k_{2}-k_{1}\right) \cdot \zeta_{4}\left(k_{3} \cdot k_{4}\right)-\left(k_{2}-k_{1}\right) \cdot k_{4}\left(k_{3} \cdot \zeta_{4}\right)\right\} \\
& +\left(\bar{u}_{1} k_{3} u_{2}\right)\left\{\left(k_{2}-k_{1}\right) \cdot k_{4}\left(\zeta_{3} \cdot \zeta_{4}\right)-\left(k_{2}-k_{1}\right) \cdot \zeta_{4}\left(k_{4} \cdot \zeta_{3}\right)\right\} \\
& +\left(\bar{u}_{1} \zeta_{4} u_{2}\right)\left\{\left(k_{2}-k_{1}\right) \cdot \zeta_{3}\left(k_{3} \cdot k_{4}\right)-\left(k_{2}-k_{1}\right) \cdot k_{3}\left(k_{4} \cdot \zeta_{3}\right)\right\} \\
& +\left(\bar{u}_{1} k_{4} u_{2}\right)\left\{\left(k_{2}-k_{1}\right) \cdot k_{3}\left(\zeta_{3} \cdot \zeta_{4}\right)-\left(k_{2}-k_{1}\right) \cdot \zeta_{3}\left(k_{3} \cdot \zeta_{4}\right)\right\} \\
& +\left(\bar{u}_{1} \gamma_{\mu \nu \rho} u_{2}\right)\left\{\left(k_{2}+k_{1}\right) \cdot \zeta_{4} k_{3}^{\mu} k_{4}^{\nu} \zeta_{3}^{\rho}-\left(k_{2}+k_{1}\right) \cdot \zeta_{3} k_{3}^{\mu} k_{4}^{\nu} \zeta_{4}^{\rho}\right. \\
& \left.\left.+\left(k_{2}+k_{1}\right) \cdot k_{4} k_{3}^{\mu} \zeta_{3}^{\nu} \zeta_{4}^{\rho}-\left(k_{2}+k_{1}\right) \cdot k_{3} k_{4}^{\mu} \zeta_{3}^{\nu} \zeta_{4}^{\rho}\right\}\right],
\end{aligned}
$$

where $u_{1}$ and $u_{2}$ denote the fermion wave functions, $\zeta_{3}$ and $\zeta_{4}$ denote the boson polarizations and $k_{j}$ denotes the $j$-th particle momentum.

3. 4 fermions:

$$
\begin{aligned}
& K_{4 \mathrm{f}}=\frac{1}{24}\left[\left\{k_{1} \cdot k_{4}+k_{2} \cdot k_{3}-k_{1} \cdot k_{3}-k_{2} \cdot k_{4}\right\} \bar{u}_{1} \gamma_{\mu} u_{2} \bar{u}_{3} \gamma^{\mu} u_{4}\right. \\
& +\left\{k_{1} \cdot k_{2}+k_{3} \cdot k_{4}-k_{1} \cdot k_{4}-k_{2} \cdot k_{3}\right\} \bar{u}_{1} \gamma_{\mu} u_{3} \bar{u}_{2} \gamma^{\mu} u_{4} \\
& \left.+\left\{k_{1} \cdot k_{3}+k_{2} \cdot k_{4}-k_{1} \cdot k_{2}-k_{3} \cdot k_{4}\right\} \bar{u}_{1} \gamma_{\mu} u_{4} \bar{u}_{2} \gamma^{\mu} u_{3}\right],
\end{aligned}
$$

where $u_{j}$ and $k_{j}$ denote the $j$-th fermion wave function and momentum, respectively.

All these kinematic factors have alternative expressions when considering the momentum conservation and the on-shell conditions. For example, using the $\gamma^{\mu}$ matrix relations in (A.2) and (A.3), relation (A.5) for commuting spinors (with $p=1$ and $p=3$ ), the on-shell conditions $k_{3} \cdot \zeta_{3}=k_{4} \cdot \zeta_{4}=\bar{u}_{2} \not k_{2}=k_{1} u_{1}=\not k_{4} k_{4}=0$, momentum conservation and introducing the Mandelstam variables (2.4), the kinematic factors in (B.5) and (B.6) may be shortly written as

$$
\begin{aligned}
K_{2 \mathrm{~b} / 2 \mathrm{f}}= & -\frac{s}{8}\left[\bar{u}_{2} \oiint_{3}\left(k_{4}+\not k_{1}\right) \oiint_{4} u_{1}\right] \\
& -\frac{t}{8}\left[2\left(\bar{u}_{2} \Varangle_{3} u_{1}\right)\left(k_{3} \cdot \zeta_{4}\right)+2\left(\bar{u}_{2} k_{4} u_{1}\right)\left(\zeta_{3} \cdot \zeta_{4}\right)-2\left(\bar{u}_{2} \oiint_{4} u_{1}\right)\left(k_{4} \cdot \zeta_{3}\right)\right]
\end{aligned}
$$

and

$$
K_{4 \mathrm{f}}=-\frac{s}{8} \bar{u}_{1} \gamma_{\mu} u_{4} \bar{u}_{2} \gamma^{\mu} u_{3}+\frac{t}{8} \bar{u}_{1} \gamma_{\mu} u_{2} \bar{u}_{4} \gamma^{\mu} u_{3}
$$

in agreement with [17], after taking into account the observations of [15] about the global factors in the 4-point amplitudes ${ }^{16}$.

The way we have chosen to write the kinematic factors in (B.4), (B.5) and (B.6) is such that the corresponding boson and/or fermion symmetry is manifest: $K_{4 \mathrm{~b}}$ is completely symmetric under any particle interchange; $K_{2 \mathrm{~b} / 2 \mathrm{f}}$ is antisymmetric under interchanges of particles 1 and 2 (the fermions) and is symmetric under interchanges of particles 3 and 4 (the bosons); $K_{4 \mathrm{f}}$ is completely antisymmetric under any particle interchange.

\footnotetext{
${ }^{16}$ Due to the different conventions in the Mandelstam variables, our formulas (B.7) and (B.8) do not look directly the same as the ones in formulas (2.6) and (2.4) of [15], but the explicit dependence of the kinematic factors in terms of the momenta $k_{j}$ is the same.
} 


\section{B.2 The 4-point effective action}

In this Appendix we deal only with the $\alpha^{\prime}$ correction terms of the effective action, that is, $S_{\alpha^{\prime} \text { corr. }}^{\text {nonael. }}$. We begin writing it as the sum of two contributions:

$$
S_{\alpha^{\prime} \text { corr. }}^{\text {nonabel. }}[A, \psi]=S_{\alpha^{\prime} \text { corr. }}^{(4)}[A, \psi]+S_{\alpha^{\prime} \text { corr. }}^{(n>4)}[A, \psi],
$$

where $S_{\alpha^{\prime} \text { corr. }}^{(4)}[A, \psi]$ contains only the quartic field terms and $S_{\alpha^{\prime} \text { corr. }}^{(n>4)}[A, \psi]$ contains the higher field terms (which are not present in the abelian theory).

The quartic field terms action in (B.9) may be written as

$$
\begin{aligned}
S_{\alpha^{\prime} \text { corr. }}^{(4)}[A, \psi]= & \iiint \int\left\{\prod_{j=1}^{4} d^{10} x_{j}\right\}\left[\frac{1}{4 !} \Gamma_{\mu_{1} \mu_{2} \mu_{3} \mu_{4}}^{(4) a_{1} a_{2} a_{3} a_{4}}\left(x_{1}, x_{2}, x_{3}, x_{4}\right) A_{a_{1}}^{\mu_{1}}\left(x_{1}\right) A_{a_{2}}^{\mu_{2}}\left(x_{2}\right) A_{a_{3}}^{\mu_{3}}\left(x_{3}\right) A_{a_{4}}^{\mu_{4}}\left(x_{4}\right)\right. \\
& +\frac{1}{2 ! \cdot 2 !} \Gamma_{\alpha_{1} \alpha_{2} \mu_{3} \mu_{4}}^{(4) a_{1} a_{2} a_{3} a_{4}}\left(x_{1}, x_{2}, x_{3}, x_{4}\right) \psi_{a_{1}}^{\alpha_{1}}\left(x_{1}\right) \psi_{a_{2}}^{\alpha_{2}}\left(x_{2}\right) A_{a_{3}}^{\mu_{3}}\left(x_{3}\right) A_{a_{4}}^{\mu_{4}}\left(x_{4}\right) \\
& \left.+\frac{1}{4 !} \Gamma_{\alpha_{1} \alpha_{2} \alpha_{3} \alpha_{4}}^{(4) a_{1} a_{2} a_{3} a_{4}}\left(x_{1}, x_{2}, x_{3}, x_{4}\right) \psi_{a_{1}}^{\alpha_{1}}\left(x_{1}\right) \psi_{a_{2}}^{\alpha_{2}}\left(x_{2}\right) \psi_{a_{3}}^{\alpha_{3}}\left(x_{3}\right) \psi_{a_{4}}^{\alpha_{4}}\left(x_{4}\right)\right]
\end{aligned}
$$

where

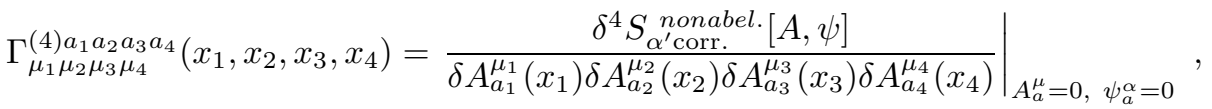

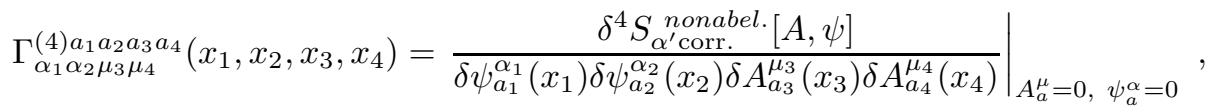

$$
\begin{aligned}
& \Gamma_{\alpha_{1} \alpha_{2} \alpha_{3} \alpha_{4}}^{(4) a_{1} a_{2} a_{3} a_{4}}\left(x_{1}, x_{2}, x_{3}, x_{4}\right)=\left.\frac{\delta^{4} S_{\alpha^{\prime} \text { corr. }}^{\text {nonabl. }}[A, \psi]}{\delta \psi_{a_{1}}^{\alpha_{1}}\left(x_{1}\right) \delta \psi_{a_{2}}^{\alpha_{2}}\left(x_{2}\right) \delta \psi_{a_{3}}^{\alpha_{3}}\left(x_{3}\right) \delta \psi_{a_{4}}^{\alpha_{4}\left(x_{4}\right)}}\right|_{A_{a}^{\mu}=0, \psi_{a}^{\alpha}=0} .
\end{aligned}
$$

The relations in (B.11) assume that the fermionic functional derivatives are being taken from the right hand-side at any moment.

Using the explicit expression of $S_{\alpha^{\prime} \text { corr. }}^{\text {nonabl. }}[A, \psi]$ in (2.10), and the identity (A.16) for the $t_{(8)}$ tensor, we obtain ${ }^{17}$ :

$$
\begin{gathered}
\Gamma_{\mu_{1} \mu_{2} \mu_{3} \mu_{4}}^{(4) a_{1} a_{2} a_{3} a_{4}}\left(x_{1}, x_{2}, x_{3}, x_{4}\right)= \\
-8 g^{2} \alpha^{\prime 2} \int d^{10} x t_{\mu_{1} \nu_{1} \mu_{2} \nu_{2} \mu_{3} \nu_{3} \mu_{4} \nu_{4}}^{(8)} \\
\mathcal{G}_{\alpha^{\prime} \text { corr. }}^{a_{1} a_{2} a_{3} a_{4}}\left(\partial_{1} \cdot \partial_{2}+\partial_{3} \cdot \partial_{4}, \partial_{1} \cdot \partial_{4}+\partial_{2} \cdot \partial_{3}, \partial_{1} \cdot \partial_{3}+\partial_{2} \cdot \partial_{4}\right) \\
\partial_{1}^{\nu_{1}} \delta^{(10)}\left(x-x_{1}\right) \partial_{2}^{\nu_{2}} \delta^{(10)}\left(x-x_{2}\right) \partial_{3}^{\nu_{3}} \delta^{(10)}\left(x-x_{3}\right) \partial_{4}^{\nu_{4}} \delta^{(10)}\left(x-x_{4}\right), \\
\Gamma_{\alpha_{1} \alpha_{2} \mu_{3} \mu_{4}}^{(4) a_{1} a_{2} a_{3} a_{4}}\left(x_{1}, x_{2}, x_{3}, x_{4}\right)= \\
i g^{2} \alpha^{\prime 2} \int d^{10} x \mathcal{G}_{\alpha^{\prime} \text { corr. }}^{a_{1} a_{2} a_{3} a_{4}}\left(\partial_{1} \cdot \partial_{2}+\partial_{3} \cdot \partial_{4}, \partial_{1} \cdot \partial_{4}+\partial_{2} \cdot \partial_{3}, \partial_{1} \cdot \partial_{3}+\partial_{2} \cdot \partial_{4}\right) \\
{\left[-\left\{\left(\gamma^{0} \gamma_{\mu_{3}}\right)_{\alpha_{1} \alpha_{2}} \partial_{\mu_{4}}^{2} \partial_{\nu}^{3} \partial_{4}^{\nu}+\left(\gamma^{0} \gamma_{\mu_{3}}\right)_{\alpha_{1} \alpha_{2}} \partial_{\nu}^{2} \partial_{\mu_{4}}^{3} \partial_{4}^{\nu}\right.\right.} \\
\left.+\left(\gamma^{0} \gamma^{\nu}\right)_{\alpha_{1} \alpha_{2}} \eta_{\mu_{3} \mu_{4}} \partial_{\rho}^{2} \partial_{\nu}^{3} \partial_{4}^{\rho}+\left(\gamma^{0} \gamma^{\nu}\right)_{\alpha_{1} \alpha_{2}} \partial_{\mu_{4}}^{2} \partial_{\nu}^{3} \partial_{\mu_{3}}^{4}\right\} \\
+\left\{\left(\gamma^{0} \gamma_{\sigma \mu_{3} \rho}\right)_{\alpha_{1} \alpha_{2}} \partial_{\mu_{4}}^{2} \partial_{3}^{\sigma} \partial_{4}^{\rho}-\left(\gamma^{0} \gamma_{\sigma \mu_{3} \mu_{4}}\right)_{\alpha_{1} \alpha_{2}} \partial_{2}^{\rho} \partial_{3}^{\sigma} \partial_{\rho}^{4}\right\} \\
\left.+\left(\begin{array}{c}
18 \text { terms obtained by antisymmetrizing on indexes }(1,2) \text { and }) \\
\text { symmetrizing on indexes }(3,4) \text { the two previous curly brackets }
\end{array}\right)\right] \\
\delta^{(10)}\left(x-x_{1}\right) \delta^{(10)}\left(x-x_{2}\right) \delta^{(10)}\left(x-x_{3}\right) \delta^{(10)}\left(x-x_{4}\right),
\end{gathered}
$$

\footnotetext{
${ }^{17}$ In all these formulas we use the notation $\partial_{j}^{\nu}=\partial / \partial x_{\nu}^{j}$.
} 


$$
\begin{aligned}
& \Gamma_{\alpha_{1} \alpha_{2} \alpha_{3} \alpha_{4}}^{(4) a_{1} a_{2} a_{3} a_{4}}\left(x_{1}, x_{2}, x_{3}, x_{4}\right)= \\
& \frac{1}{6} g^{2} \alpha^{\prime 2} \int d^{10} x \mathcal{G}_{\alpha^{\prime} \text { corr. }}^{a_{1} a_{2} a_{3} a_{4}}\left(\partial_{1} \cdot \partial_{2}+\partial_{3} \cdot \partial_{4}, \partial_{1} \cdot \partial_{4}+\partial_{2} \cdot \partial_{3}, \partial_{1} \cdot \partial_{3}+\partial_{2} \cdot \partial_{4}\right) \\
& \quad\left[\left(\gamma^{0} \gamma^{\mu}\right)_{\alpha_{1} \alpha_{2}}\left(\gamma^{0} \gamma_{\mu}\right)_{\alpha_{3} \alpha_{4}} \partial_{2}^{\nu} \partial_{\nu}^{4}\right. \\
& +(23 \text { terms obtained by antisymmetrizing on indexes }(1,2,3,4))] \\
& \quad \delta^{(10)}\left(x-x_{1}\right) \delta^{(10)}\left(x-x_{2}\right) \delta^{(10)}\left(x-x_{3}\right) \delta^{(10)}\left(x-x_{4}\right),
\end{aligned}
$$

where $\mathcal{G}_{\alpha^{\prime} \text { corr. }}^{a_{1} a_{2} a_{3} a_{4}}(s, t, u)$ is given in $(2.11)$.

By definition (see (B.11)), all 4-point functions have the appropriate boson and/or fermion symmetry, as was commented in the previous subsection in the case of the kinematic factors. This boson and/or fermion symmetry can be checked directly in the expressions (B.12), (B.13) and (B.14).

\section{B.3 The scattering amplitudes}

¿From the previous 4-point functions, the corresponding 4-point scattering amplitudes are calculated, in our conventions, as

$$
\mathcal{A}_{\alpha^{\prime} \text { corr. }}^{(4)}=-i(2 \pi)^{10} \delta^{(10)}\left(k_{1}+k_{2}+k_{3}+k_{4}\right) \phi_{1}^{p_{1}} \phi_{2}^{p_{2}} \phi_{3}^{p_{3}} \phi_{4}^{p_{4}} \Gamma_{p_{1} p_{2} p_{3} p_{4}}^{(4) a_{1} a_{2} a_{3} a_{4}}\left(k_{1}, k_{2}, k_{3}, k_{4}\right)
$$

where

$$
\begin{aligned}
&(2 \pi)^{10} \delta^{(10)}\left(k_{1}+k_{2}+k_{3}+k_{4}\right) \Gamma_{p_{1} p_{2} p_{3} p_{4}}^{(4) a_{2} a_{2} a_{3} a_{4}}\left(k_{1}, k_{2}, k_{3}, k_{4}\right)= \\
& \iiint \int\left\{\prod_{j=1}^{4} d^{10} x_{j} e^{i k_{j} \cdot x_{j}}\right\} \Gamma_{p_{1} p_{2} p_{3} p_{4}}^{(4) a_{1} a_{2} a_{3} a_{4}}\left(x_{1}, x_{2}, x_{3}, x_{4}\right) .
\end{aligned}
$$

In (B.15), $p_{j}$ and $\phi_{j}^{p_{j}}$ denote, respectively, the $j$-th vector (spinor) index and the $j$-th boson polarization vector (fermion wave function), according to the specific scattering process.

Upon substituting (B.12), (B.13) and (B.14) in (B.16) and then in (B.15), all three cases can be summarized as

$$
\mathcal{A}_{\alpha^{\prime} \text { corr. }}^{(4)}=8 i g^{2} \alpha^{\prime 2}(2 \pi)^{10} \delta^{(10)}\left(k_{1}+k_{2}+k_{3}+k_{4}\right) \mathcal{G}_{\alpha^{\prime} \text { corr. }}^{a_{1} a_{2} a_{3} a_{4}}(s, t, u) K^{(4)},
$$

where, for each scattering process, $K^{(4)}$ is exactly the same kinematic factor given in (B.4), (B.5) and (B.6).

So, substituting (B.2) and (B.17) in (B.1) we finally see that our nonabelian 4-point effective action in (2.8), (2.9) and (2.10), reproduces the known 4-particle scattering amplitudes of Open Superstring theory:

$$
\begin{array}{rl}
\mathcal{A}^{(4)}=8 & i g^{2} \alpha^{\prime 2}(2 \pi)^{10} \delta^{(10)}\left(k_{1}+k_{2}+k_{3}+k_{4}\right) \times \\
\times\left[\frac{\Gamma\left(-\alpha^{\prime} s\right) \Gamma\left(-\alpha^{\prime} t\right)}{\Gamma\left(1-\alpha^{\prime} s-\alpha^{\prime} t\right)}\left\{\operatorname{tr}\left(\lambda^{a_{1}} \lambda^{a_{2}} \lambda^{a_{3}} \lambda^{a_{4}}\right)+\operatorname{tr}\left(\lambda^{a_{4}} \lambda^{a_{3}} \lambda^{a_{2}} \lambda^{a_{1}}\right)\right\}\right. \\
\quad+\frac{\Gamma\left(-\alpha^{\prime} t\right) \Gamma\left(-\alpha^{\prime} u\right)}{\Gamma\left(1-\alpha^{\prime} t-\alpha^{\prime} u\right)}\left\{\operatorname{tr}\left(\lambda^{a_{1}} \lambda^{a_{3}} \lambda^{a_{2}} \lambda^{a_{4}}\right)+\operatorname{tr}\left(\lambda^{a_{4}} \lambda^{a_{2}} \lambda^{a_{3}} \lambda^{a_{1}}\right)\right\} \\
\left.\quad+\frac{\Gamma\left(-\alpha^{\prime} u\right) \Gamma\left(-\alpha^{\prime} s\right)}{\Gamma\left(1-\alpha^{\prime} u-\alpha^{\prime} s\right)}\left\{\operatorname{tr}\left(\lambda^{a_{1}} \lambda^{a_{2}} \lambda^{a_{4}} \lambda^{a_{3}}\right)+\operatorname{tr}\left(\lambda^{a_{3}} \lambda^{a_{4}} \lambda^{a_{2}} \lambda^{a_{1}}\right)\right\}\right] \cdot K^{(4)} .
\end{array}
$$




\section{C. $f$ - $f$ part of the $\mathcal{O}\left(\alpha^{\prime 5}\right)$ action}

The $\mathcal{S}_{(5), f-f}$ action in $(2.28)$ is given by

$$
\begin{aligned}
& \mathcal{S}_{(5), f-f}= \\
& \int d^{10} x f_{b}^{a_{1} a_{2} b} f_{b}^{a_{3} a_{4}}\left\{\left[D_{\kappa} D_{\tau} D_{\lambda} F_{a_{1} \mu \nu} D^{\kappa} D^{\tau} F_{a_{2}}^{\nu \rho} D^{\lambda} F_{a_{3} \rho \sigma} F_{a_{4}}^{\sigma \mu}\right.\right. \\
& -\frac{1}{4} D_{\kappa} D_{\tau} D_{\lambda} F_{a_{1} \mu \nu} D^{\kappa} D^{\tau} F_{a_{2}}^{\mu \nu} D^{\lambda} F_{a_{3} \rho \sigma} F_{a_{4}}^{\rho \sigma}+2 i D_{\kappa} D_{\tau} D_{\lambda} \bar{\psi}_{a_{1}} \gamma^{\nu} D^{\kappa} D^{\tau} D_{\rho} \psi_{a_{2}} D^{\lambda} F_{a_{3} \nu \mu} F_{a_{4}}^{\mu \rho} \\
& \text { - } i D_{\kappa} D_{\tau} D_{\lambda} \bar{\psi}_{a_{1}} \gamma^{\mu \nu \rho} D^{\kappa} D^{\tau} D^{\sigma} \psi_{a_{2}} D^{\lambda} F_{a_{3} \mu \nu} F_{a_{4} \rho \sigma} \\
& \left.-\frac{1}{3} D_{\kappa} D_{\tau} D_{\lambda} \bar{\psi}_{a_{1}} \gamma_{\mu} D^{\kappa} D^{\tau} D_{\nu} \psi_{a_{2}} D^{\lambda} \bar{\psi}_{a_{3}} \gamma^{\mu} D^{\nu} \psi_{a_{4}}\right] \\
& +\left[D_{\kappa} D_{\tau} D_{\lambda} F_{\mu \nu} a_{1} F_{a_{2}}^{\nu \rho} D^{\lambda} F_{a_{3} \rho \sigma} D^{\kappa} D^{\tau} F_{a_{4}}^{\sigma \mu}\right. \\
& -\frac{1}{4} D_{\kappa} D_{\tau} D_{\lambda} F_{a_{1} \mu \nu} F_{a_{2}}^{\mu \nu} D^{\lambda} F_{a_{3} \rho \sigma} D^{\kappa} D^{\tau} F_{a_{4}}^{\rho \sigma}+2 i D_{\kappa} D_{\tau} D_{\lambda} \bar{\psi}_{a_{1}} \gamma^{\nu} D_{\rho} \psi_{a_{2}} D^{\lambda} F_{a_{3} \nu \mu} D^{\kappa} D^{\tau} F_{a_{4}}^{\mu \rho} \\
& \text { - } i D_{\kappa} D_{\tau} D_{\lambda} \bar{\psi}_{a_{1}} \gamma^{\mu \nu \rho} D^{\sigma} \psi_{a_{2}} D^{\lambda} F_{a_{3} \mu \nu} D^{\kappa} D^{\tau} F_{a_{4} \rho \sigma} \\
& \left.-\frac{1}{3} D_{\kappa} D_{\tau} D_{\lambda} \bar{\psi}_{a_{1}} \gamma_{\mu} D_{\nu} \psi_{a_{2}} D^{\lambda} \bar{\psi}_{a_{3}} \gamma^{\mu} D^{\kappa} D^{\tau} D^{\nu} \psi_{a_{4}}\right] \\
& +\left[D_{\kappa} D_{\tau} D_{\lambda} F_{a_{1} \mu \nu} F_{a_{2}}^{\nu \rho} D^{\kappa} D^{\tau} D^{\lambda} F_{a_{3} \rho \sigma} F_{a_{4}}^{\sigma \mu}\right. \\
& -\frac{1}{4} D_{\kappa} D_{\tau} D_{\lambda} F_{a_{1} \mu \nu} F_{a_{2}}^{\mu \nu} D^{\kappa} D^{\tau} D^{\lambda} F_{a_{3} \rho \sigma} F_{a_{4}}^{\rho \sigma}+2 i D_{\kappa} D_{\tau} D_{\lambda} \bar{\psi}_{a_{1}} \gamma^{\nu} D_{\rho} \psi_{a_{2}} D^{\kappa} D^{\tau} D^{\lambda} F_{a_{3} \nu \mu} F_{a_{4}}^{\mu \rho} \\
& \text { - } i D_{\kappa} D_{\tau} D_{\lambda} \bar{\psi}_{a_{1}} \gamma^{\mu \nu \rho} D^{\sigma} \psi_{a_{2}} D^{\kappa} D^{\tau} D^{\lambda} F_{a_{3} \mu \nu} F_{a_{4} \rho \sigma} \\
& \left.-\frac{1}{3} D_{\kappa} D_{\tau} D_{\lambda} \bar{\psi}_{a_{1}} \gamma_{\mu} D_{\nu} \psi_{a_{2}} D^{\kappa} D^{\tau} D^{\lambda} \bar{\psi}_{a_{3}} \gamma^{\mu} D^{\nu} \psi_{a_{4}}\right] \\
& +\int d^{10} x f^{a_{1} a_{3} b} f_{b}^{a_{2} a_{4}}\left\{\left[D_{\kappa} D_{\tau} D_{\lambda} F_{a_{1} \mu \nu} D^{\kappa} D^{\tau} D^{\lambda} F_{a_{2}}^{\nu \rho} F_{a_{3} \rho \sigma} F_{a_{4}}^{\sigma \mu}\right.\right. \\
& -\frac{1}{4} D_{\kappa} D_{\tau} D_{\lambda} F_{a_{1} \mu \nu} D^{\kappa} D^{\tau} D^{\lambda} F_{a_{2}}^{\mu \nu} F_{a_{3} \rho \sigma} F_{a_{4}}^{\rho \sigma}+2 i D_{\kappa} D_{\tau} D_{\lambda} \bar{\psi}_{a_{1}} \gamma^{\nu} D^{\kappa} D^{\tau} D^{\lambda} D_{\rho} \psi_{a_{2}} F_{a_{3} \nu \mu} F_{a_{4}}^{\mu \rho} \\
& \text { - } i D_{\kappa} D_{\tau} D_{\lambda} \bar{\psi}_{a_{1}} \gamma^{\mu \nu \rho} D^{\kappa} D^{\tau} D^{\lambda} D^{\sigma} \psi_{a_{2}} F_{a_{3} \mu \nu} F_{a_{4} \rho \sigma} \\
& \left.-\frac{1}{3} D_{\kappa} D_{\tau} D_{\lambda} \bar{\psi}_{a_{1}} \gamma_{\mu} D^{\kappa} D^{\tau} D^{\lambda} D_{\nu} \psi_{a_{2}} \bar{\psi}_{a_{3}} \gamma^{\mu} D^{\nu} \psi_{a_{4}}\right] \\
& +\left[D_{\kappa} D_{\tau} D_{\lambda} F_{a_{1} \mu \nu} D^{\lambda} F_{a_{2}}^{\nu \rho} F_{a_{3} \rho \sigma} D^{\kappa} D^{\tau} F_{a_{4}}^{\sigma \mu}\right. \\
& -\frac{1}{4} D_{\kappa} D_{\tau} D_{\lambda} F_{a_{1} \mu \nu} D^{\lambda} F_{a_{2}}^{\mu \nu} F_{a_{3} \rho \sigma} D^{\kappa} D^{\tau} F_{a_{4}}^{\rho \sigma}+2 i D_{\kappa} D_{\tau} D_{\lambda} \bar{\psi}_{a_{1}} \gamma^{\nu} D^{\lambda} D_{\rho} \psi_{a_{2}} F_{a_{3} \nu \mu} D^{\kappa} D^{\tau} F_{a_{4}}^{\mu \rho} \\
& \text { - } i D_{\kappa} D_{\tau} D_{\lambda} \bar{\psi}_{a_{1}} \gamma^{\mu \nu \rho} D^{\lambda} D^{\sigma} \psi_{a_{2}} F_{a_{3} \mu \nu} D^{\kappa} D^{\tau} F_{a_{4} \rho \sigma} \\
& \left.-\frac{1}{3} D_{\kappa} D_{\tau} D_{\lambda} \bar{\psi}_{a_{1}} \gamma_{\mu} D^{\lambda} D_{\nu} \psi_{a_{2}} \bar{\psi}_{a_{3}} \gamma^{\mu} D^{\kappa} D^{\tau} D^{\nu} \psi_{a_{4}}\right] \\
& +\left[D_{\kappa} D_{\tau} D_{\lambda} F_{a_{1} \mu \nu} D^{\lambda} F_{a_{2}}^{\nu \rho} D^{\kappa} D^{\tau} F_{a_{3} \rho \sigma} F_{a_{4}}^{\sigma \mu}\right. \\
& -\frac{1}{4} D_{\kappa} D_{\tau} D_{\lambda} F_{a_{1} \mu \nu} D^{\lambda} F_{a_{2}}^{\mu \nu} D^{\kappa} D^{\tau} F_{a_{3} \rho \sigma} F_{a_{4}}^{\rho \sigma}+2 i D_{\kappa} D_{\tau} D_{\lambda} \bar{\psi}_{a_{1}} \gamma^{\nu} D^{\lambda} D_{\rho} \psi_{a_{2}} D^{\kappa} D^{\tau} F_{a_{3} \nu \mu} F_{a_{4}}^{\mu \rho} \\
& \text { - } i D_{\kappa} D_{\tau} D_{\lambda} \bar{\psi}_{a_{1}} \gamma^{\mu \nu \rho} D^{\lambda} D^{\sigma} \psi_{a_{2}} D^{\kappa} D^{\tau} F_{a_{3} \mu \nu} F_{a_{4} \rho \sigma} \\
& \left.\left.-\frac{1}{3} D_{\kappa} D_{\tau} D_{\lambda} \bar{\psi}_{a_{1}} \gamma_{\mu} D^{\lambda} D_{\nu} \psi_{a_{2}} D^{\kappa} D^{\tau} \bar{\psi}_{a_{3}} \gamma^{\mu} D^{\nu} \psi_{a_{4}}\right]\right\}
\end{aligned}
$$

We have directly used eq. (2.27) to derive this expression, without looking for any simplification when using integration by parts, equations of motion or the identities in Appendix A. So it may 
well happen that the number of terms in this action reduces if these last considerations are taken into account.

\section{References}

[1] M. Born and L. Infeld, Foundations of the new field theory, Proc. Roy. Soc. Lond. A144 (1934) 425.

[2] E.S. Fradkin and A.A. Tseytlin, Nonlinear electrodynamics from quantized strings, Phys. Lett. B 163 (1985) 123.

[3] A.A. Tseytlin, Vector field effective action in the open superstring theory, Nucl. Phys. B 276 (1986) 391.

[4] D.J. Gross and E. Witten, Superstring modifications of Einstein's equations, Nucl. Phys. B 277 (1986) 1.

[5] Y. Kitazawa, Effective lagrangian for the open superstring from a 5-point function, Nucl. Phys. B 289 (1987) 599.

[6] O. D. Andreev and A. A. Tseytlin, Partition function representation for the open superstring effective action: cancellation of Möbius infinities and derivative corrections to Born-Infeld lagrangian, Nucl. Phys. B 311 (1988) 205.

[7] E. Witten, Bound states of strings and p-branes, Nucl. Phys. B 460 (1996) 35, [hep-th/9510135].

[8] M. Cederwall, A. von Gussich, B.E.W. Nilsson, P. Sundell and A. Westerberg, The Dirichlet Super Three-Brane in Ten-Dimensional Type IIB Supergravity, Nucl. Phys. B 490 (1997) 163, [hep-th/9610148] .

[9] M. Cederwall, A. von Gussich, B.E.W. Nilsson, P. Sundell and A. Westerberg, The Dirichlet Super-p-Branes in Ten-Dimensional Type IIA and IIB Supergravity, Nucl. Phys. B 480 (1997) 179 [hep-th/9611159].

[10] E. Bergshoeff and P.K. Townsend, Super-D-branes, Nucl. Phys. B 490 (1997) 145, [hep-th/9611173].

[11] M. Aganagic, C. Popsescu and J. H. Schwarz, Gauge-invariant and gauge-fixed D-brane actions, Nucl. Phys. B 495 (1997) 99, [hep-th/9612080].

[12] N. Wyllard, Derivative corrections to the D-brane Born-Infeld action: non geodesic embeddings and the Seiberg-Witten map, J. High Energy Phys. 08 (2001) 027 [hep-th/0107185].

[13] N. Wyllard, Derivative corrections to D-brane actions with constant background fields, Nucl. Phys. B 598 (2001) 247 [hep-th/0008125].

[14] A.A. Tseytlin, On non-abelian generalization of Born-Infeld action in string theory, Nucl. Phys. B 501 (1997) 41 [hep-th/9701125].

[15] E. A. Bergshoeff, A. Bilal, M. de Roo and A. Sevrin, Supersymmetric nonabelian Born-Infeld revisited, J. High Energy Phys. 07 (2001) 029 [hep-th/0105274].

[16] A. Bilal, Higher-derivative corrections to the non-abelian Born-Infeld action, Nucl. Phys. B 618 (2001) 21 [hep-th/0106062].

[17] J. H. Schwarz, Superstring Theory, Phys. Rept. 89 (1982) 233.

[18] F. Brandt, F. Machado and R. Medina, The open superstring 5-point amplitude revisited, J. High Energy Phys. 07 (2002) 071 [hep-th/0208121].

[19] P. Koerber and A. Sevrin, The non-abelian Born-Infeld action through order $\alpha^{\prime 3}$, J. High Energy Phys. 10 (2001) 003 [hep-th/0108169]; Testing the ${\alpha^{\prime}}^{3}$ term in the non-abelian open superstring effective action, J. High Energy Phys. 09 (2001) 009 [hep-th/0109030].

[20] A. Refolli, A. Santambrogio, N. Terzi and D. Zanon, $F^{5}$ contributions to the non-abelian Born-Infeld action from a supersymmetric Yang-Mills five-point function, Nucl. Phys. B 613 (2001) 64 [hep-th/0105277]. 
[21] D. T. Grasso, Higher order contributions to the effective action of $N=4$ super Yang-Mills, J. High Energy Phys. 0211 (2002) 012 [hep-th/0210146].

[22] L. De Fossé, P. Koerber and A. Sevrin, The uniqueness of the nonabelian Born-Infeld action, Nucl. Phys. B 603 (2001) 413 [hep-th/0103015].

[23] P. Koerber and A. Sevrin, The non-abelian D-brane effective action through order $\alpha^{\prime 4}$, J. High Energy Phys. 10 (2002) 046 [hep-th/0208044]

[24] A. Collinucci, M. De Roo and M.G.C. Eenink, Supersymmetric Yang-Mills theory at order $\alpha^{\prime 3}, J^{.}$ High Energy Phys. 06 (2002) 024 [hep-th/0205150].

[25] A. Collinucci, M. De Roo and M.G.C. Eenink, Derivative corrections in 10-dimensional super-Maxwell theory, J. High Energy Phys. 0301 (2003) 039 [hep-th/0212012].

[26] M. Cederwall, B.E.W. Nilsson and D. Tsimpis, The structure of maximally supersymmetric Yang-Mills theory: constraining higher-order corrections, J. High Energy Phys. 06 (2001) 034 [hep-th/0102009].

[27] M. Cederwall, B.E.W. Nilsson and D. Tsimpis, D=10 super Yang-Mills at $\alpha^{\prime 2}$, J. High Energy Phys. 07 (2001) 042 [hep-th/0104236].

[28] J. M. Drummond, P.J. Heslop, P. S. Howe and S.F. Kerstan, Integral invariants in N=4 SYM and the effective action for coincident D-branes, J. High Energy Phys. 0308 (2003) 016 [hep-th/0305202].

[29] M. De Roo and M.G.C. Eenink, The effective action for the 4-point functions in abelian open superstring theory, J. High Energy Phys. 0308 (2003) 036 [hep-th/0307211].

[30] M. T. Grisaru, A. E. van de Ven and D. Zanon, Two-dimensional supersymmetric sigma models on Ricci flat Kahler manifolds are not finite, Nucl. Phys. B 277 (1986) 388; Four Loop Divergences For The N=1 Supersymmetric Nonlinear Sigma Model In Two-Dimensions, Nucl. Phys. B 277 (1986) 409.

[31] Y. Kikuchi, C. Marzban and Y. J. Ng, Heterotic string modifications of Einstein's and Yang-Mills' actions, Phys. Lett. B 176 (1986) 57.

[32] Y. Cai, C. A. Núñez, Heterotic string covariant amplitudes and low-energy effective action, Nucl. Phys. B 287 (1987) 279.

[33] D. J. Gross and J. H. Sloan, The Quartic Effective Action For The Heterotic String, Nucl. Phys. B 291 (1987) 41.

[34] R. R. Metsaev and A. A. Tseytlin, On loop corrections to string theory effective actions, Nucl. Phys. B 298 (1988) 109.

[35] J. G. Russo, Construction of SL(2,Z) invariant amplitudes in type IIB superstring theory, Nucl. Phys. B 535 (1998) 116 [hep-th/9802090].

[36] M. B. Green, H. h. Kwon and P. Vanhove, Two loops in eleven dimensions, Phys. Rev. D 61 (2000) 104010 [hep-th/9910055].

[37] G. Arfken, Mathematical Methods for Physicists, third edition, San Diego, USA: Academic Press, 1985.

[38] S. Nagaoka, Fluctuation Analysis of Non-abelian Born-Infeld Action in the Background Intersecting D-branes, hep-th/0307232.

[39] J. Polchinski, String theory, vol. 2: Superstring theory and beyond, Cambridge, UK: Univ. Pr. 1998.

[40] M. B. Green and S. Sethi, Supersymmetry constraints on type IIB supergravity, Phys. Rev. D 59 (1999) 046006 [hep-th/9808061].

[41] M. B. Green and M. Gutperle, Effects of D-instantons Nucl. Phys. B 498 (1997) 195 [hep-th/9701093] 